\title{
Some remarkable new plethystic operators in the theory of Macdonald polynomials
}

\author{
Francois Bergeron*, Adriano Garsia ${ }^{\dagger}$, \\ Emily Sergel Leven ${ }^{\ddagger}$, AND GuOCE XIN ${ }^{\S}$
}

\begin{abstract}
In the 90's a collection of plethystic operators were introduced in [2], [7] and [8] to solve some representation-theoretical problems arising from the theory of Macdonald polynomials. This collection was enriched in the research that led to the results which appeared in [5], [6] and [9]. However since some of the identities resulting from these efforts were eventually not needed, this additional work remained unpublished. As a consequence of very recent publications [4], [11], [19], [20], [21], a truly remarkable expansion of this theory has taken place. However most of this work has appeared in a language that is virtually inaccessible to practitioners of algebraic combinatorics. Yet, these developments have led to a variety of new conjectures in [3] in the combinatorics and symmetric function theory of Macdonald polynomials. The present work results from an effort to obtain in an elementary and accessible manner all the background necessary to construct the symmetric function side of some of these new conjectures. It turns out that the above mentioned unpublished results provide precisely the tools needed to carry out this project to its completion.
\end{abstract}

\section{Introduction}

Our main actors in this development are the operators $D_{k}$ introduced in [8], whose action on a symmetric function $F[X]$ is defined by setting

$$
D_{k} F[X]=\left.F\left[X+\frac{M}{z}\right] \sum_{i \geq 0}(-z)^{i} e_{i}[X]\right|_{z^{k}} \quad(\text { with } M=(1-t)(1-q)) .
$$

These operators generate an algebra $\mathcal{A}$ of symmetric function operators with remarkable properties. To state them we need some preliminary observations and definitions. Let us denote by $\Lambda$ the space of symmetric functions in the

arXiv: 1405.0316

*Partially supported by NSERC-Canada.

${ }^{\dagger}$ Partially supported by NSF grant DMS 1362160.

†Supported by NSF grant DGE 1144086 .

${ }^{\S}$ Partially supported by NSFC(11171231). 
infinite alphabet $X=\left\{x_{1}, x_{2}, x_{3}, \ldots\right\}$ and by $\Lambda^{=d}$ the subspace of homogeneous symmetric functions of degree $d$. It is easy to see from 1 that if $F[X] \in \Lambda^{=d}$ then $D_{k} F[X] \in \Lambda^{=d+k}$. Thus $\mathcal{A}$ is clearly a graded algebra. What is surprising is that $\mathcal{A}$ is in fact bi-graded by simply assigning the generators $D_{k}$ bi-degree $(1, k)$.

To make this more precise consider first $\mathcal{D}=\left\{D_{0}, D_{1}, D_{2}, D_{3}, \ldots\right\}$ as an infinite alphabet, and denote by $\mathcal{L}[\mathcal{D}]$ the linear span of words in $\mathcal{D}$. Now, given this bi-grading of the letters of $\mathcal{D}$, every element $\Pi \in \mathcal{L}[\mathcal{D}]$ has a natural decomposition

$$
\Pi=\sum_{(u, v)} \Pi_{u, v}
$$

where $\Pi_{u, v}$ denotes the portion of $\Pi$ which is a linear combination of words in $\mathcal{D}$ of total bi-degree $(u, v)$. To show that $\mathcal{A}$ is bi-graded it is necessary and sufficient to prove that $\Pi$, as an operator, acts by zero on $\Lambda$ if and only if all the $\Pi_{u, v}$ act by zero. This is one of the very first things we will prove about $\mathcal{A}$.

The connection of $\mathcal{A}$ to the above mentioned developments is that it gives a concrete realization of a proper subspace of the elliptic Hall algebra studied by Schiffmann and Vasserot in [20], [21] and [19]. In particular it contains a distinguished family of operators $\left\{Q_{u, v}\right\}$ of bi-degree given by their index that play a central role in the above mentioned conjectures. For a co-prime bi-degree their construction is so simple that we need only illustrate it in a special case.

For instance, to obtain $Q_{3,5}$ we start by drawing the $3 \times 5$ lattice square with its diagonal (the line $(0,0) \rightarrow(3,5)$, as shown in the adjacent figure), we then look for the lattice point $(a, b)$ that is closest to and below the diagonal. In this case $(a, b)=(2,3)$. This yields the decomposition $(3,5)=$ $(2,3)+(1,2)$ and we set

$$
Q_{3,5}=\frac{1}{M}\left[Q_{1,2}, Q_{2,3}\right]=\frac{1}{M}\left(Q_{1,2} Q_{2,3}-Q_{2,3} Q_{1,2}\right) .
$$

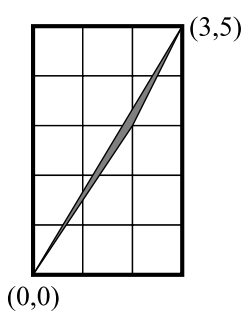

We must next work precisely in the same way with the $2 \times 3$ rectangle and, as indicated in the adjacent figure, obtain the decomposition $(2,3)=(1,1)+(1,2)$ and set

$$
Q_{2,3}=\frac{1}{M}\left[Q_{1,2}, Q_{1,1}\right]=\frac{1}{M}\left(Q_{1,2} Q_{1,1}-Q_{1,1} Q_{1,2}\right) .
$$

Now, in this case, we are done, since it turns out that we may set

$$
Q_{1, k}=D_{k}
$$


In particular by combining 2,3 and 4 we obtain

$$
Q_{3,5}=\frac{1}{M^{2}}\left(D_{2} D_{2} D_{1}-2 D_{2} D_{1} D_{2}+D_{1} D_{2} D_{2}\right) .
$$

In the general co-prime case $(m, n)$, the precise definition is based on an elementary number theoretical lemma that characterizes the closest lattice point $(a, b)$ below the line $(0,0) \rightarrow(m, n)$. We then let $(c, d)=(m, n)-(a, b)$ and set

$$
\operatorname{Split}(m, n)=(a, b)+(c, d) .
$$

This given, we recursively define

(6) $\quad Q_{m, n}= \begin{cases}\frac{1}{M}\left[Q_{c, d}, Q_{a, b}\right] & \text { if } m>1 \text { and } \operatorname{Split}(m, n)=(a, b)+(c, d) \\ D_{n} & \text { if } m=1 .\end{cases}$

Our next task is to define the operators $Q_{u, v}$ for any non co-prime pair $(u, v)$. It will be convenient here and after to write such a pair in the form $(k m, k n)$ with $(m, n)$ co-prime and $k>1$ the $g c d$ of the pair. The problem is that in this case there are exactly $k$ lattice points, closest to the diagonal of the rectangle $k m \times k n$, as we can clearly see in the following display, where we illustrate the case $(m, n)=(3,2)$ and $k=4$.
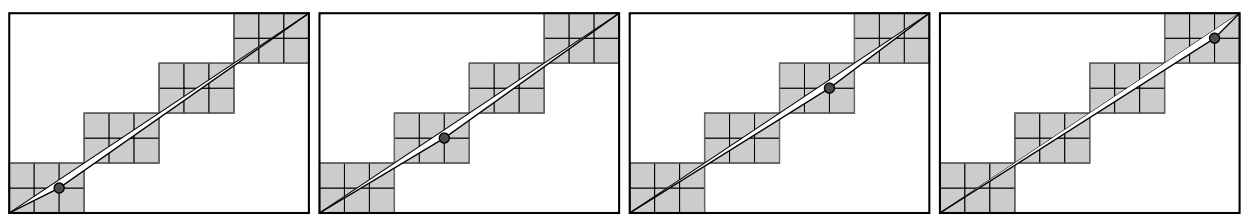

We see that there are 4 ways here to "split" the vector $(0,0) \rightarrow(4 \times$ $3,4 \times 2$ ) by choosing a closest lattice point below the diagonal. Namely:

$$
(12,8)=(2,1)+(10,7)=(5,3)+(7,5)=(8,5)+(4,3)=(11,7)+(1,1) .
$$

This given, which of the following bracketings should we choose to construct $Q_{4 \times 3,4 \times 2}$ ?

$$
\left[Q_{10,7}, Q_{2,1}\right], \quad\left[Q_{7,5}, Q_{5,3}\right], \quad\left[Q_{4,3}, Q_{8,5}\right], \quad\left[Q_{1,1}, Q_{11,7}\right] .
$$

The answer is simple: any one will do, since all four bracketings give the same operator. This is one of the many identities we need to establish for the operators $Q_{m, n}$. In fact all the pairs $(a, b)$ and $(c, d)$ obtained by splitting 
a pair $(k m, k n)$, with $(a, b)$ one of the closest lattice points to the segment $(0,0) \rightarrow(k m, k n)$, are necessarily co-prime. Our original idea was to prove first the auxiliary identities needed to construct the operators $Q_{0, n}$ then obtain all the other needed identities as images of the auxiliary identities, under the action of the modular group $G=S L_{2}[Z]$ on the operators $Q_{m, n}$. In the realization of this plan, the operators $Q_{n, n}$ were more convenient to work with.

More precisely, for a given element $\left[\begin{array}{ll}a & c \\ b & d\end{array}\right]$ we will show that we can set

$$
\left[\begin{array}{ll}
a & c \\
b & d
\end{array}\right] Q_{m, n}=Q_{a m+c n, b m+d n}
$$

by proving that two generators of $G$ preserve all the relations satisfied by the operators $D_{k}$.

As we will see, this is made possible by means of a very elementary, but surprisingly powerful tool, in algebraic combinatorics which has come to be called the Stanton-Stembridge symmetrization trick (the SSS trick briefly).

By combining the above mentioned auxiliary identities with the action of $G$ we will also be naturally led to the construction of a variety of new additional operators. More precisely there is one operator for each symmetric function $G[X]$, homogeneous of degree $k$ and each co-prime pair $(m, n)$. The resulting operator, which will be denoted " $\mathbf{G}_{k m, k n}$," turns out to have a variety of surprising properties. In fact, computer exploration led to the discovery (in [3]) that in many instances the symmetric polynomial $\mathbf{G}_{k m, k n}(-1)^{k(n+1)}$ has a conjectured combinatorial interpretation as an enumerator of certain families of "rational" parking functions.

One of the most surprising contributions to this branch of algebraic combinatorics is a recent deep result [17] of Andrei Negut giving a relatively simple but powerful constant term expression for the action of the operators $Q_{m, n}$. The reader is referred to the findings concerning the Negut formula that are presented in [3] for the reasons we used the word "powerful" in this context. Here it has been one of our priorities to give a straight-forward proof of Negut's formula using only tools developed in the present treatment of the subject. In our third and final section we present the various results obtained in this effort. Our main result there is a proof that the validity of the Negut formula is equivalent to the statement that a certain quite elementary and completely explicit rational function symmetrizes to zero. It will be seen that this is but another beautiful consequence of the SSS trick. This leads to a computer proof of the Negut formula in a variety of cases. Moreover, our proof makes it quite clear why and how the so-called "shuffle algebra" 
naturally arises in the present context. In fact, it should be straightforward to extend the machinery used in the proof of the above result to obtain a proof that, under appropriate definitions, the shuffle algebra is isomorphic to the algebra generated by the operators $D_{k}$. Our presentation terminates with a proof of the Negut formula under the specialization at $t=1 / q$.

\section{Acknowledgment}

We cannot overemphasize here the importance of the contribution of Eugene Gorsky and Andrei Negut to the present developments. Without their efforts at translating their results [11], [18], [17] and results of SchiffmannVasserot [20], [21], [19] in a language understandable to us, this writing would not have been possible.

\section{Notation and auxiliary identities}

In dealing with symmetric function identities, especially those arising in the theory of Macdonald polynomials, it is convenient and often indispensable to use plethystic notation. This device has a straightforward definition which can be verbatim implemented in MAPLE or MATHEMATICA. We simply set for any expression $E=E\left(t_{1}, t_{2}, \ldots\right)$ and any symmetric function $F$

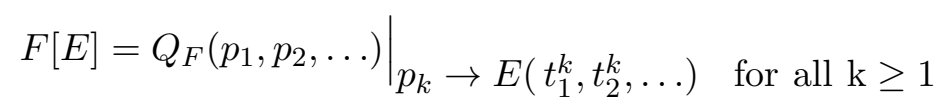

where $Q_{F}$ is the polynomial yielding the expansion of $F$ in terms of the power basis. A paradoxical but necessary property of plethystic substitutions is that 7 requires $p_{k}[-E]=-p_{k}[E]$. This notwithstanding, we will also need to carry out ordinary changes of signs. To distinguish the latter from the "plethystic" minus sign, we will carry out the "ordinary" sign change by multiplying our expressions by a new variable " $\epsilon$ " which, outside of the plethystic bracket, is replaced by -1 . Thus we have

$$
p_{k}[\epsilon E]=\epsilon^{k} p_{k}[E]=(-1)^{k} p_{k}[E] .
$$

In particular we see that, with this notation, it follows that for any expression $E$ and any symmetric function $F$ we may write

$$
(\omega F)[E]=F[-\epsilon E]
$$

where, as customary, " $\omega$ " denotes the involution that interchanges the elementary and homogeneous symmetric function bases. 
It will be also good to remind the reader here that many symmetric function identities can be considerably simplified by means of the " $\Omega$ " notation. For a general expression $E=E\left(t_{1}, t_{2}, \cdots\right)$ we simply set

$$
\Omega[E]=\exp \left(\sum_{k \geq 1} \frac{p_{k}[E]}{k}\right)=\exp \left(\sum_{k \geq 1} \frac{E\left(t_{1}^{k}, t_{2}^{k}, \cdots\right)}{k}\right) .
$$

In particular we see that for $X=x_{1}+x_{2}+\cdots$

$$
\Omega[z X]=\sum_{m \geq 0} z^{m} h_{m}[X]
$$

and for $M=(1-t)(1-q)$ we have

$$
\Omega[-u M]=\frac{(1-u)(1-q t u)}{(1-t u)(1-q u)} .
$$

As in Macdonald's [16], for each (french) Ferrers diagram of a partition $\mu$, and a lattice cell $c \in \mu$ we have four parameters $l=l_{\mu}(c), l^{\prime}=l_{\mu}^{\prime}(c)$, $a=a_{\mu}(c)$ and $a^{\prime}=a_{\mu}^{\prime}(c)$ called leg, coleg, arm and coarm which give the number of lattice cells of $\mu$ strictly north, south, east and west of $c$. Denoting by $\mu^{\prime}$ the conjugate of $\mu$, the basic ingredients we need to keep in mind here are

$$
\begin{gathered}
n(\mu)=\sum_{i=1}^{l(\mu)}(i-1) \mu_{i}, \quad w_{\mu}(q, t)=\prod_{c \in \mu}\left(q^{a_{\mu}(c)}-t^{l_{\mu}(c)+1}\right)\left(t^{l_{\mu}(c)}-q^{a_{\mu}(c)+1}\right), \\
T_{\mu}=t^{n(\mu)} q^{n\left(\mu^{\prime}\right)}, \quad B_{\mu}(q, t)=\sum_{c \in \mu} t^{l_{\mu}^{\prime}(c)} q^{a_{\mu}^{\prime}(c)}, \quad M=(1-t)(1-q) .
\end{gathered}
$$

Let us recall that the Hall scalar product is defined by setting

$$
\left\langle p_{\lambda}, p_{\mu}\right\rangle=z_{\mu} \chi(\lambda=\mu)^{1}
$$

where $z_{\mu}$ gives the order of the stabilizer of a permutation with cycle structure $\mu$.

The Macdonald polynomials we work with here are the unique [7] symmetric function basis $\left\{\widetilde{H}_{\mu}[X ; q, t]\right\}_{\mu}$ which is upper triangularly (in dominance order) related to the modified Schur basis $\left\{s_{\lambda}\left[\frac{X}{t-1}\right]\right\}_{\lambda}$ and satisfies the orthogonality condition

\footnotetext{
${ }^{1}$ Here and after we let $\chi(\mathbf{A})=1$ if $\mathbf{A}$ is true and $\chi(\mathbf{A})=0$ if $\mathbf{A}$ is false.
} 


$$
\left\langle\widetilde{H}_{\lambda}, \widetilde{H}_{\mu}\right\rangle_{*}=\chi(\lambda=\mu) w_{\mu}(q, t),
$$

where $\langle,\rangle_{*}$ denotes a deformation of the Hall scalar product, defined by setting

$$
\left\langle p_{\lambda}, p_{\mu}\right\rangle_{*}=(-1)^{|\mu|-l(\mu)} \prod_{i}\left(1-t^{\mu_{i}}\right)\left(1-q^{\mu_{i}}\right) z_{\mu} \chi(\lambda=\mu) .
$$

We will use here the operator $\nabla$ introduced in [1] by setting

$$
\nabla \widetilde{H}_{\mu}[X ; q, t]=T_{\mu} \widetilde{H}_{\mu}[X ; q, t] .
$$

We also set for any symmetric function $F[X]$

$$
D_{k}^{*} F[X]=\left.F\left[X-\frac{\widetilde{M}}{z}\right] \sum_{i \geq 0} z^{i} h_{i}[X]\right|_{z^{k}} \quad(\text { with } \widetilde{M}=(1-1 / t)(1-1 / q))
$$

It will be convenient to use the symbol " $F$ " to denote the operator "multiplication" by a symmetric function $F[X]$. These families of operators were intensively studied in the $90^{\prime} s$ (see [2] and [8]) where they gave rise to a variety of conjectures, some of which are still open.

In particular it is shown in [8] that the operators $D_{k}, D_{k}^{*}, \nabla$ and the modified Macdonald polynomials $\widetilde{H}_{\mu}[X ; q, t]$ are related by the following identities.

\section{Proposition 1.1}

The operators $D_{0}, D_{0}^{*}$ and $\nabla$ are all self-adjoint with respect to the *scalar product. Moreover for $k \geq 1$, the operators $p_{k}, D_{k}$ and $D_{k}^{*}$ are $*$-scalar product adjoints to $M(-1)^{k-1} p_{k}^{\perp},(-1)^{k} D_{-k}$ and $(-q t)^{k} D_{-k}^{*}$ respectively. We also have

$$
\begin{array}{cccc}
\text { (i) } & D_{0} \widetilde{H}_{\mu}=-D_{\mu}(q, t) \widetilde{H}_{\mu} & (i)^{*} & D_{0}^{*} \widetilde{H}_{\mu}=-D_{\mu}(1 / q, 1 / t) \widetilde{H}_{\mu} \\
\text { (ii) } & D_{k} \underline{e}-\underline{e} D_{k}=M D_{k+1} & (i i)^{*} & D_{k}^{*} \underline{e}-\underline{e} D_{k}^{*}=-\widetilde{M} D_{k+1}^{*} \\
\text { (iii) } & \nabla \underline{e} \nabla^{-1}=-D_{1} & (\text { iii })^{*} & \nabla D_{1}^{*} \nabla^{-1}=\underline{e} \\
\text { (iv) } & \nabla^{-1} e_{1}^{\perp} \nabla=\frac{1}{M} D_{-1} & (i v)^{*} & \nabla^{-1} D_{-1}^{*} \nabla=-\widetilde{M} e_{1}^{\perp}
\end{array}
$$

with $e_{1}^{\perp}$ the Hall scalar product adjoint of multiplication by $e_{1}, \widetilde{M}=(1-$ $1 / t)(1-1 / q)$ and

$$
D_{\mu}(q, t)=M B_{\mu}(q, t)-1 .
$$


We should mention that recursive applications of $15(i i)$ and $(i i)^{*}$ give

$$
\begin{aligned}
& \text { a) } D_{k}=\frac{1}{M^{k}} \sum_{r=0}^{k}\left(\begin{array}{l}
k \\
r
\end{array}\right)(-1)^{r} \underline{e}_{1}^{r} D_{0} \underline{e}_{1}^{k-r} \\
& \text { b) } D_{k}^{*}=\frac{1}{\bar{M}^{k}} \sum_{r=0}^{k}\left(\begin{array}{l}
k \\
r
\end{array}\right)(-1)^{k-r} \underline{e}_{1}^{r} D_{0}^{*} \underline{e}_{1}^{k-r} .
\end{aligned}
$$

For future use, it will be convenient to set

$$
\begin{aligned}
& \text { a) } \Phi_{k}=\nabla D_{k} \nabla^{-1} \quad \text { and } \\
& \text { b) } \Psi_{k}=-(q t)^{1-k} \nabla D_{k}^{*} \nabla^{-1} \text {. }
\end{aligned}
$$

This given we have

\section{Theorem 1.1}

The operators $\Phi_{k}$ and $\Psi_{k}$ are uniquely determined by the following recursions
a) $\Phi_{k+1}=\frac{1}{M}\left[D_{1}, \Phi_{k}\right]$
and
b) $\Psi_{k+1}=\frac{1}{M}\left[\Psi_{k}, D_{1}\right]$

and initial conditions
a) $\Phi_{1}=\frac{1}{M}\left[D_{1}, D_{0}\right]$
and
b) $\Psi_{1}=-\underline{e}_{1}$.

\section{Proof}

Note first that, using 15 (ii) and (iii), the definition in 18 a) for $k=1$ gives

$$
\Phi_{1}=\frac{1}{M} \nabla\left(D_{0} e_{1}-e_{1} D_{0}\right) \nabla^{-1}=\frac{1}{M}\left(D_{0}\left(-D_{1}\right)-\left(-D_{1}\right) D_{0}\right)
$$

which is another way of writing $20 \mathrm{a}$ ). The definition in $18 \mathrm{~b}$ ) and $15(\mathrm{iii})^{*}$ give $20 \mathrm{~b}$ ).

Next, conjugating 15 (ii) by $\nabla$ and using 15 (iii) immediately gives 19 a). Finally note that, since $\widetilde{M}=M / q t$ it follows that $15(i i)^{*}$ may be rewritten as

$$
(q t)^{1-k} D_{k}^{*} e_{1}-e_{1}(q t)^{1-k} D_{k}^{*}=-M(q t)^{1-k-1} D_{k+1}^{*}
$$

and $19 \mathrm{~b}$ ) then follows by conjugating both sides by $\nabla$ and using 15 (iii).

The next identity plays a crucial role in the present development.

\section{Theorem 1.2}

For $a, b \in Z$ with $a+b>0$ and any symmetric function $F[X]$, we have 


$$
\frac{1}{M}\left(D_{a} D_{b}^{*}-D_{b}^{*} D_{a}\right) F[X]=\frac{(q t)^{b}}{1-q t} h_{a+b}[X(1 / q t-1)] F[X] .
$$

A proof of the general identity that includes 21 is given in the Appendix. As a corollary we obtain.

\section{Theorem 1.3}

The operators $\Phi_{k}$ and $\Psi_{k}$ defined in 18 satisfy the following identity, when $a, b$ are positive integers with sum equal to $n$ :

$$
\frac{1}{M}\left[\Psi_{b}, \Phi_{a}\right]=\frac{q t}{1-q t} \nabla \underline{h}_{n}\left[X\left(\frac{1}{q t}-1\right)\right] \nabla^{-1}
$$

\section{Proof}

The identity in 21 essentially says that under the given hypotheses the operator $\frac{1}{M}\left(D_{b}^{*} D_{a}-D_{a} D_{b}^{*}\right)$ acts as multiplication by the symmetric function $\frac{(q t)^{b}}{q t-1} h_{n}[X(1 / q t-1)]$. Thus with our notational conventions 21 may be rewritten as

$$
-\frac{(q t)^{1-b}}{M}\left(D_{b}^{*} D_{a}-D_{a} D_{b}^{*}\right)=\frac{q t}{1-q t} \underline{h}_{n}[X(1 / q t-1)] .
$$

Conjugating both sides by $\nabla$ and using 18 a) and b) gives 22 .

Next it is important to keep in mind the following identity which expresses the action of a sequence of $D_{k}$ operators on a symmetric function $F[X]$.

\section{Proposition 1.2}

$$
\begin{array}{r}
D_{a_{m}} \cdots D_{a_{1}} F[X]=F\left[X+\sum_{i=1}^{m} \frac{M}{z_{i}}\right] \prod_{i=1}^{m} \Omega\left[-z_{i} X\right] \frac{1}{\prod_{i=1}^{m} z_{i}^{a_{i}}} \\
\times\left.\prod_{1 \leq i<j \leq m} \Omega\left[-M z_{i} / z_{j}\right]\right|_{z_{1}^{0} z_{2}^{0} \cdots z_{m}^{0}}
\end{array}
$$

\section{Proof}

It suffices to see what happens when we use 7 a) twice:

$$
\begin{aligned}
D_{a_{2}} D_{a_{1}} F[X] & =\left.D_{a_{2}} F\left[X+\frac{M}{z_{1}}\right] \Omega\left[-z_{1} X\right]\right|_{z_{1}^{a_{1}}} \\
& =\left.F\left[X+\frac{M}{z_{1}}+\frac{M}{z_{2}}\right] \Omega\left[-z_{1}\left(X+\frac{M}{z_{2}}\right)\right] \Omega\left[-z_{2} X\right]\right|_{z_{1}^{a_{1}} z_{2}^{a_{2}}} \\
& =\left.F\left[X+\frac{M}{z_{1}}+\frac{M}{z_{2}}\right] \Omega\left[-z_{1} X\right] \Omega\left[-z_{2} X\right] \Omega\left[-M z_{1} / z_{2}\right]\right|_{z_{1}^{a_{1}} z_{2}^{a_{2}}}
\end{aligned}
$$


To give a precise general definition of the $Q$ operators we need the following elementary number theoretical fact that characterizes the closest lattice point $(a, b)$ below the line $(0,0) \rightarrow(m, n)$.

\section{Proposition 1.3}

For any pair of co-prime integers $m, n>1$ there is a unique pair $a, b$ satisfying the following three conditions

$$
\text { (1) } 1 \leq a \leq m-1, \quad \text { (2) } 1 \leq b \leq n-1, \quad \text { (3) } m b+1=n a
$$

In particular, setting $(c, d)=(m, n)-(a, b)$ we will write for $m, n>1$

$$
\operatorname{Split}(m, n)=(a, b)+(c, d)
$$

and otherwise set

$$
\begin{aligned}
& \text { a) } \operatorname{Split}(1, n)=(1, n-1)+(0,1) \\
& \text { b) } \operatorname{Split}(m, 1)=(1,0)+(m-1,1) .
\end{aligned}
$$

Moreover it follows from our construction that the pairs $(a, b)$ and $(c, d)$ are also co-prime.

\section{Proof}

When $m, n>1$ the lattice point that is closest to and strictly below the diagonal of the $m \times n$ lattice rectangle must be the unique element of the set

$$
\left\{\left(i,\left\lfloor i \frac{n}{m}\right\rfloor\right): 1 \leq i \leq m-1\right\}
$$

that minimizes the difference

$$
\epsilon_{i}=i \frac{n}{m}-\left\lfloor i \frac{n}{m}\right\rfloor .
$$

In fact, the co-primality of $m, n$ assures that all these differences are distinct. So the distance minimizer is clearly unique. Next note that if we set

$$
k_{i}=m \epsilon_{i}=i n-m\left\lfloor i \frac{n}{m}\right\rfloor
$$

then $k_{i}$ is an integer in the interval

$$
1 \leq k_{i} \leq m-1
$$

Since all the $k_{i}$ must be distinct and there are altogether $m-1$ of them, exactly one of them must be equal to 1 . If $k_{a}=1$ then the minimizing point is $(a, b)$ with $b=\left\lfloor a \frac{n}{m}\right\rfloor$, and 28 for $i=a$ reduces to 


$$
1=a n-m b .
$$

This proves (1) and (3) of 24 and (2) is then an immediate consequence of (1) and $b=\left\lfloor a \frac{n}{m}\right\rfloor$. Finally, 26 is simply due to the fact that in either of these two cases the closest point can be easily identified. The co-primality of $(a, b)$ is immediate, since if $(a, b)=\left(k a^{\prime}, k b^{\prime}\right)$ for some $k>1$ then $\left(a^{\prime}, b^{\prime}\right)$ would be closer to the diagonal $(0,0) \rightarrow(m, n)$. The co-primality of $(c, d)$ holds for the identical reason.

We are now in a position to give the definition of the operators $Q_{m, n}$ that is more suitable for theoretical purposes.

\section{Definition 1.1} set

$$
Q_{m, n}=\frac{1}{M}\left[Q_{c, d}, Q_{a, b}\right]
$$

with base cases
a) $Q_{1,0}=D_{0}$ and
b) $Q_{0,1}=-\underline{e}_{1}$.

It is easy to see from Proposition 1.3 that recursive applications of 30 will eventually lead to an expression for $Q_{m, n}$ as a polynomial in the non commutative operators $D_{0}$ and $\underline{e}_{1}$.

For computer programming purposes the following alternate recursive construction is considerably more efficient since, via 23 , it gives all these operators a plethystic form.

\section{Theorem 1.4}

For any pair of co-prime $m, n$ we have

$$
Q_{m, n}= \begin{cases}\frac{1}{M}\left[Q_{c, d}, Q_{a, b}\right] & \text { if } m>1 \text { and } \operatorname{Split}(m, n)=(a, b)+(c, d) \\ D_{n} & \text { if } m=1\end{cases}
$$

\section{Proof}

Since at each application of the Split operation for $m, n>1$ we have both $a \leq m-1$ and $c \leq n-1$, we will eventually reach the point in the recursion expressed by 30 where $m=1$ or $n=1$. In the first case, 26 a) takes over and the identity in 15 (ii) inductively assures that $Q_{1, n}=D_{n}$. In fact, in the base case we have, (by 15 (ii) for $k=0$ )

$$
Q_{1,1}=\frac{1}{M}\left[Q_{0,1}, Q_{1,0}\right]=\frac{1}{M}\left[-e_{1}, D_{0}\right]=\frac{1}{M}\left[D_{0}, e_{1}\right]=D_{1}
$$

In case $n=1$ and $m>1$ then $26 \mathrm{~b}$ ) takes over, yielding 


$$
Q_{m, 1}=\frac{1}{M}\left[Q_{m-1,1}, Q_{1,0}\right]=\frac{1}{M}\left[Q_{m-1,1}, D_{0}\right]
$$

Here the base case is reached when $m=2$ yielding

$$
Q_{2,1}=\frac{1}{M}\left[Q_{1,1}, Q_{1,0}\right]=\frac{1}{M}\left[D_{1}, D_{0}\right]
$$

We terminate this section with the following truly surprising and remarkably basic identity in our development.

\section{Proposition $\mathbf{1 . 6}$}

For any co-prime pair $m, n$ we have

$$
Q_{m+n, n}=\nabla Q_{m, n} \nabla^{-1}
$$

\section{Proof}

We proceed by induction on the size of $\min \{m, n\}$. Suppose first that $m, n \geq 2$ and

$$
\operatorname{Split}(m, n)=(a, b)+(c, d) .
$$

Suppose inductively that we have

$$
Q_{a+b, b}=\nabla Q_{a, b} \nabla^{-1} \quad \text { and } \quad Q_{c+d, d}=\nabla Q_{c, d} \nabla^{-1}
$$

From 36 and Proposition 1.3 it follows that
(1) $1 \leq a \leq m-1$,
(2) $1 \leq b \leq n-1$,
(3) $b m+1=n a$

Adding $n b$ to both sides of (3) gives

$$
\left(3^{\prime}\right) \quad b(m+n)+1=n(a+b),
$$

while from (1) and (2) it follows that

$$
\left(1^{\prime}\right) 1 \leq a+b \leq m+n-1, \quad\left(2^{\prime}\right) 1 \leq b \leq n-1,
$$

But $\left(1^{\prime}\right),\left(2^{\prime}\right),\left(3^{\prime}\right)$, by Proposition 1.3, imply that

$$
\operatorname{Split}(m+n, n)=(a+b, b)+(c+d, d) .
$$

This gives

$$
Q_{m+n, n}=\frac{1}{M}\left[Q_{c+d, d}, Q_{a+b, b}\right]
$$

and from 37 we derive that 


$$
Q_{m+n, n}=\nabla Q_{m, n} \nabla^{-1}
$$

completing the induction.

We are left with checking the equality in the cases where $m \leq 1$ or $n \leq 1$. This brings us to the two identities (38)
a) $\operatorname{Split}(1, n)=(1, n-1)+(0,1)$,
b) $\operatorname{Split}(m, 1)=(1,0)+(m-1,1)$.

The common base case is $(1,1)$. There we must show that

$$
Q_{1,1}=\nabla Q_{0,1} \nabla^{-1} \text {. }
$$

But by $31 \mathrm{~b}$ ) and 32 this is

$$
D_{1}=-\nabla e_{1} \nabla^{-1}
$$

which is 15 (iii). We can thus proceed by induction in each case. Now for case a) we have

$$
Q_{1, n}=\frac{1}{M}\left[Q_{0,1}, Q_{1, n-1}\right] .
$$

Assuming that the result is true for $n-1$ gives

$$
\nabla Q_{1, n} \nabla^{-1}=\frac{1}{M}\left[\nabla Q_{0,1} \nabla^{-1}, \nabla Q_{1, n-1} \nabla^{-1}\right]=\frac{1}{M}\left[Q_{1,1}, Q_{n, n-1}\right] .
$$

Since $\operatorname{Split}(n+1, n)=(n, n-1)+(1,1)$ we see that $\frac{1}{M}\left[Q_{1,1}, Q_{n, n-1}\right]=$ $Q_{n+1, n}$. This completes the induction in case a). Proceeding again by induction in case b) we get

$$
\nabla Q_{m, 1} \nabla^{-1}=\frac{1}{M}\left[\nabla Q_{m-1,1} \nabla^{-1}, \nabla Q_{1,0} \nabla^{-1}\right]=\frac{1}{M}\left[Q_{m, 1}, Q_{1,0}\right]
$$

and again $38 \mathrm{~b}$ ) gives $\nabla Q_{m, 1} \nabla^{-1}=Q_{m+1,1}$ completing the induction and our proof.

\section{The action of $\mathrm{SL}_{2}[\mathrm{Z}]$ on the algebra generated by the operators $D_{k}$}

To extend the definition of the $Q$ operators to non-coprime pairs of indices we need to make use of the action of $S L_{2}[Z]$ on the operators $Q_{m, n}$. More precisely, for $\left[\begin{array}{ll}a & c \\ b & d\end{array}\right] \in S L_{2}[Z]$ and any co-prime pair $(m, n)$ we want

$$
\left[\begin{array}{ll}
a & c \\
b & d
\end{array}\right] Q_{m, n}=Q_{a m+c n, b n+d n}
$$


For this it is sufficient to justify setting

$$
N Q_{m, n}=Q_{m+n, n} \quad \text { and } \quad S Q_{m, n}=Q_{m, n+m}
$$

for the generators

$$
N=\left[\begin{array}{ll}
1 & 1 \\
0 & 1
\end{array}\right] \quad \text { and } \quad S=\left[\begin{array}{ll}
1 & 0 \\
1 & 1
\end{array}\right]
$$

Since every operator $Q_{m, n}$ is a polynomial in the operators $D_{k}=Q_{1, k}$ we will define this action on the algebra generated by the $D_{k}$ by setting

$$
N\left(D_{k_{1}} D_{k_{2}} \cdots D_{k_{r}}\right)=Q_{1+k_{1}, k_{1}} Q_{1+k_{2}, k_{2}} \cdots Q_{1+k_{r}, k_{r}}
$$

and

$$
S\left(D_{k_{1}} D_{k_{2}} \cdots D_{k_{r}}\right)=Q_{1, k_{1}+1} Q_{1, k_{2}+1} \cdots Q_{1, k_{r}+1}
$$

For this action to be well defined it is necessary and sufficient that if any polynomial in the $D_{k}$ that acts by zero on symmetric functions, then it has an image under $N$ and $S$ which also acts by zero. Now it happens that this fact can be proved by elementary means.

To begin, notice that the identity 35 allows us to rewrite 41 as

$$
N\left(D_{k_{1}} D_{k_{2}} \cdots D_{k_{r}}\right)=\nabla\left(D_{k_{1}} D_{k_{2}} \cdots D_{k_{r}}\right) \nabla^{-1}
$$

This implies the desired property for the action of $N$, since any symmetric function operator that acts by zero has an image under conjugation by $\nabla$ which also acts by zero.

To prove that $S$ has the desired property we will make use of a simple observation which has come to be referred to as the Stanton-Stembridge symmetrization trick. This trick uses symmetrization to prove that certain constant terms are 0. Stanton, Stembridge, and Zeilberger used these techniques in special cases before Zeilberger formally stated the general trick in [24]. A partial history of these early uses can be found in [23].

\section{SSS trick}

For a Laurent polynomial $F\left(z_{1}, z_{2}, \ldots, z_{m}\right)$ we have

$$
\left.F\left(z_{1}, z_{2}, \ldots, z_{m}\right)\right|_{z_{1}^{0} z_{2}^{0} \cdots z_{m}^{0}}=0
$$

if and only if 


$$
\left.\operatorname{Sym}_{m} F\left(z_{1}, z_{2}, \ldots, z_{m}\right)\right|_{z_{1}^{0} z_{2}^{0} \cdots z_{m}^{0}}=0
$$

where "Sym $m_{m}$ " is the idempotent that symmetrizes with respect to the variables $z_{1}, z_{2}, \ldots, z_{m}$.

It is important to notice that consequently this is also valid when $F$ is a formal power series in other variables with coefficients Laurent polynomials in $z_{1}, z_{2}, \ldots, z_{m}$. The surprising circumstance is that quite often the identity in 45 turns out to be a consequence of the more encompassing identity

$$
\operatorname{Sym}_{m} F\left(z_{1}, z_{2}, \ldots, z_{m}\right)=0
$$

which is sometimes easier to prove than 45. A beautiful example of this type of circumstance is given by the following result which is crucial in our development.

Here and after it will be convenient to use $Z_{k}$ as an abbreviation for the alphabet $z_{1}, z_{2}, \ldots z_{k}$.

\section{Theorem 2.1}

Suppose that $F P_{k}\left(Z_{k}\right)$ (for each $1 \leq k \leq m$ ) is formal power series in other variables with coefficients which are Laurent polynomials in $z_{1}, z_{2}, \ldots$, $z_{k}$. Then, for all symmetric functions $F[X]$ we have

$$
\left.\sum_{k=1}^{m}\left(F\left[X+\sum_{i=1}^{k} \frac{M}{z_{i}}\right] \Omega\left[-Z_{k} X\right] F P_{k}\left(Z_{k}\right) \prod_{1 \leq i<j \leq k} \Omega\left[-M z_{i} / z_{j}\right]\right)\right|_{z_{1}^{0} z_{2}^{0} \cdots z_{m}^{0}}=0
$$

if and only if

$$
\operatorname{Sym}_{k}\left(F P_{k}\left(Z_{k}\right) \prod_{1 \leq i<j \leq k} \Omega\left[-M z_{i} / z_{j}\right]\right)=0 \quad(\text { for } 1 \leq k \leq m) .
$$

In particular it follows that the operator $\mathbf{V}=\sum_{k=1}^{m} \mathbf{V}_{k}$ with $\mathbf{V}_{k}=$ $\sum_{a} c_{a_{1}, a_{2}, \ldots, a_{k}}^{(k)} D_{a_{k}} \cdots D_{a_{2}} D_{a_{1}}$ acts by zero on symmetric polynomials if and only if, setting

$$
\Pi_{\mathbf{V}_{k}}\left(Z_{k}\right)=\sum_{a} c_{a_{1}, a_{2}, \ldots, a_{k}} \frac{1}{z_{1}^{a_{1}} z_{2}^{a_{2} \cdots z_{k}^{a_{k}}}}
$$

we have

$$
\operatorname{Sym}_{k}\left(\Pi_{\mathbf{V}_{k}}\left(Z_{k}\right) \prod_{1 \leq i<j \leq k} \Omega\left[-M z_{i} / z_{j}\right]\right)=0 .
$$




\section{Proof}

Notice that since for any variable $u$ we have $\Omega[-u M]=1+$ $\sum_{r \geq 1} u^{r} h_{r}[-M]$ it follows that for any $1 \leq k \leq m$

$$
\prod_{1 \leq i<j \leq k} \Omega\left[-M z_{i} / z_{j}\right]
$$

is a formal power series in $q, t$ with coefficients Laurent polynomials in $z_{1}, z_{2}, \ldots, z_{k}$. Likewise the expression

$$
F\left[X+\sum_{i=1}^{k} \frac{M}{z_{i}}\right] \Omega\left[-Z_{k} X\right]=F\left[X+\sum_{i=1}^{k} \frac{M}{z_{i}}\right] \prod_{i=1}^{k} \Omega\left[-z_{i} X\right]
$$

may be viewed as a formal power series in the variables in $X$ and $q, t$ with coefficients Laurent polynomials in $z_{1}, z_{2}, \ldots, z_{k}$. Thus the SSS trick applies and we can derive from 46 and the $S_{k}$ symmetry of the expression in 50 that we will have 46 if and only if

$$
\left.\sum_{k=1}^{m} F\left[X+\sum_{i=1}^{k} \frac{M}{z_{i}}\right] \Omega\left[-Z_{k} X\right] G_{k}\left[Z_{k}\right]\right|_{z_{1}^{0} z_{2}^{0} \cdots z_{m}^{0}}=0
$$

where for convenience we have set

$$
G_{k}\left[Z_{k}\right]=\operatorname{Sym}_{k}\left(F P_{k}\left(Z_{k}\right) \prod_{1 \leq i<j \leq k} \Omega\left[-M z_{i} / z_{j}\right]\right) .
$$

In particular we can immediately see that 47 implies 46 . We must next show that the converse is also true. To carry this out it will be convenient to set

This given, note that the identity

$$
\frac{1}{z_{1}}+\frac{1}{z_{2}}+\cdots+\frac{1}{z_{k}}=Z_{k}^{(-1)}
$$

$$
p_{a}\left[M Z_{k}^{(-1)}\right]=p_{a}\left[X+M Z_{k}^{(-1)}\right]-p_{a}[X] \quad(\text { for all } a \geq 1)
$$

gives for $\lambda=\left(\lambda_{1}, \lambda_{2}, \ldots, \lambda_{l}\right)$

$$
\begin{aligned}
p_{\lambda}\left[M Z_{k}^{(-1)}\right] & =\prod_{i=1}^{l}\left(p_{\lambda_{i}}\left[X+M Z_{k}^{(-1)}\right]-p_{\lambda_{i}}[X]\right) \\
& =\sum_{S \subseteq\{1,2, \ldots l\}} \prod_{i \in S} p_{\lambda_{i}}\left[X+M Z_{k}^{(-1)}\right] \prod_{i \in\{1,2, \ldots l\}-S}\left(-p_{\lambda_{i}}[X]\right) .
\end{aligned}
$$

Using the fact that $p_{\lambda}\left[M Z_{k}^{(-1)}\right]=p_{\lambda}[M] p_{\lambda}\left[Z_{k}^{(-1)}\right]$, we may write 


$$
p_{\lambda}\left[Z_{k}^{(-1)}\right]=\sum_{\mu \preceq \lambda} p_{\mu}\left[X+M Z_{k}^{(-1)}\right] c_{\lambda, \mu}[X]
$$

where "§" means that all the parts of $\mu$ are parts of $\lambda$, and, more importantly, the coefficients $c_{\lambda, \mu}[X]$ do not depend on $k$. Thus a multiple use of 51 with $F=p_{\mu}$ for all $\mu \preceq \lambda$ gives

$$
\left.\sum_{k=1}^{m} p_{\lambda}\left[Z_{k}^{(-1)}\right] \Omega\left[-Z_{k} X\right] G_{k}\left[Z_{k}\right]\right|_{z_{1}^{0} z_{2}^{0} \cdots z_{m}^{0}}=0 .
$$

Since $\left\{p_{\lambda}[X]\right\}_{\lambda}$ is a symmetric function basis it follows from this that for all symmetric functions $F[X]$ we must also have

$$
\left.\sum_{k=1}^{m} F\left[Z_{k}^{(-1)}\right] \Omega\left[-Z_{k} X\right] G_{k}\left[Z_{k}\right]\right|_{z_{1}^{0} z_{2}^{0} \cdots z_{m}^{0}}=0
$$

Now notice that for all $k<m$ we have $e_{m}\left[Z_{k}^{(-1)}\right]=0$. It follows from this that setting $F[X]=e_{m}^{l}[X]$ for any $l>1$ but otherwise arbitrary we must have

$$
\left.\Omega\left[-Z_{m} X\right] G_{m}\left[Z_{m}\right]\right|_{z_{1}^{l} z_{2}^{l} \cdots z_{m}^{l}}=0 .
$$

Now the expansion $\Omega\left[-Z_{m} X\right]=\sum_{\lambda} m_{\lambda}\left[Z_{m}\right] h_{\lambda}[-X]$ together with the fact that (for $X$ an infinite alphabet) the collection $\left\{h_{\lambda}[-X]\right\}_{\lambda}$ is a symmetric function basis (thus independent) allows us to conclude that for arbitrary $\lambda$ we must have

$$
\left.m_{\lambda}\left[Z_{m}\right] G_{m}\left[Z_{m}\right]\right|_{z_{1}^{l} z_{2}^{l} \cdots z_{m}^{l}}=0 .
$$

It follows from our hypotheses that $G_{m}\left[Z_{m}\right]$ is a formal power series in other variables with coefficients Laurent polynomials in $z_{1}, z_{2}, \ldots, z_{m}$. Thus if $P\left[Z_{m}\right]$ is any one of these coefficients, from 52 we derive that we must also have

$$
\left.m_{\lambda}\left[Z_{m}\right] P\left[Z_{m}\right]\right|_{z_{1}^{l} z_{2}^{l} \cdots z_{m}^{l}}=0 .
$$

We claim that the arbitrariness of $\lambda$ and $l$ forces the vanishing of $P\left[Z_{m}\right]$. To see this note that we may make the substitution

$$
m_{\lambda}\left[Z_{m}\right]=\sum_{\lambda(p)=\lambda} z_{1}^{p_{1}} z_{2}^{p_{2}} \cdots z_{m}^{p_{m}}
$$


where " $\lambda(p)=\lambda$ " means that the non-zero parts of the weak composition $p$ rearrange to the parts of $\lambda$, and obtain

$$
\left.\sum_{\lambda(p)=\lambda} P\left[Z_{m}\right]\right|_{z_{1}^{l-p_{1}} z_{2}^{l-p_{2}} \cdots z_{m}^{l-p_{m}}}=0 .
$$

Since the symmetry of $G_{m}\left[Z_{m}\right]$ in $z_{1}, z_{2}, \ldots, z_{m}$ implies that also $P\left[Z_{m}\right]$ is symmetric in $z_{1}, z_{2}, \ldots, z_{m}$, all the above coefficients must be the same. This implies that

$$
\left.P\left[Z_{m}\right]\right|_{z_{1}^{l-p_{1}} z_{2}^{l-p_{2} \ldots z_{m}^{l-p_{m}}}}=0 \quad(\text { for } \lambda(p)=\lambda) .
$$

But the arbitrariness of $l$ and $\lambda$ gives that we have

$$
\left.\left.P\left[Z_{m}\right]\right|_{z_{1}^{q_{1}} z_{2}^{q_{2} \ldots z_{m}^{q_{m}}}}=0 \quad \text { (for all integral vectors } q=\left(q_{1}, q_{2}, \ldots, q_{n}\right)\right)
$$

Thus $P\left[Z_{m}\right]$ must identically vanish as asserted. Since this holds true for every coefficient of $G_{m}\left[Z_{m}\right]$ we are led to the conclusion that

$$
G_{m}\left[Z_{m}\right]=0
$$

This not only proves the special case $k=m$ of 47 but sets us up for an induction argument on $m$ with base case $m=1$ which is also a particular subcase of the case we have just dealt with. Our proof is thus complete.

As a corollary we obtain

\section{Theorem 2.2}

An operator $\mathbf{V}=\sum_{k=1}^{m} \mathbf{V}_{k}$ with

$$
\mathbf{V}_{k}=\sum_{a} c_{a_{1}, a_{2}, \ldots, a_{k}}^{(k)} D_{a_{k}} \cdots D_{a_{2}} D_{a_{1}}
$$

acts by zero on symmetric polynomials if and only if each of the operators $S \mathbf{V}_{k}$ acts by zero.

\section{Proof} have

By Theorem $1.1 \mathbf{V}$ acts by zero if and only if for each $1 \leq k \leq m$ we

$$
\operatorname{Sym}_{k}\left(\Pi_{\mathbf{V}_{k}}\left(z_{1}, z_{2}, \ldots, z_{k}\right) \prod_{1 \leq i<j \leq k} \Omega\left[-M z_{i} / z_{j}\right]\right)=0
$$

and $S \mathbf{V}_{k}$ acts by zero if and only if 


$$
\operatorname{Sym}_{k}\left(\Pi_{S \mathbf{V}_{k}}\left(z_{1}, z_{2}, \ldots, z_{k}\right) \prod_{1 \leq i<j \leq k} \Omega\left[-M z_{i} / z_{j}\right]\right)=0 .
$$

But from the definition in 42 it follows that

$$
\Pi_{S \mathbf{V}_{k}}\left(z_{1}, z_{2}, \ldots, z_{k}\right)=\frac{\Pi_{\mathbf{V}}\left(z_{1}, z_{2}, \ldots, z_{k}\right)}{z_{1} z_{2} \cdots z_{k}},
$$

so we see that 53 and 54 are equivalent identities.

Combining this with the identity in 43 and Theorem 1.4 we can now state

\section{Theorem 2.3}

The identities in 41 and 42 define an action of the group $G$ on the algebra generated by the operators $D_{k}$, with the property that for all $\left[\begin{array}{ll}a & c \\ b & d\end{array}\right] \in G$ we have

$$
\left[\begin{array}{ll}
a & c \\
b & d
\end{array}\right] Q_{m, n}=Q_{a m+c n, b n+d n}
$$

In particular this action preserves all the relations satisfied by the operators $Q_{m, n}$ for $(m, n)$ co-prime, such as (32) and (35).

We have now all we need to define the operators $Q_{k m, k n}$. To begin we have the following basic consequence of Theorem 1.3.

\section{Theorem 2.4}

For any $k \geq 1$ we have $Q_{k+1, k}=\Phi_{k}$ and $Q_{k-1, k}=\Psi_{k}$. In particular, for all pairs $a, b$ of positive integers with sum equal to $n$ it follows that

$$
\frac{1}{M}\left[Q_{b+1, b}, Q_{a-1, a}\right]=\frac{q t}{1-q t} \nabla \underline{h}_{n}\left[X\left(\frac{1}{q t}-1\right)\right] \nabla^{-1} .
$$

\section{Proof}

In view of $18 \mathrm{a}$ ), the first equality is a special instance of 35 . To prove the second equality, by Theorem 1.3, we only need show that the operators $Q_{k-1, k}$ satisfy the same recursions and base cases as the $\Psi_{k}$ operators. To begin, note that since $\operatorname{Split}(k, k+1)=(1,1)+(k-1, k)$ it follows that

$$
Q_{k, k+1}=\frac{1}{M}\left[Q_{k-1, k}, Q_{1,1}\right]=\frac{1}{M}\left[Q_{k-1, k}, D_{1}\right],
$$

which is $19 \mathrm{~b}$ ) for $Q_{k, k+1}$. Furthermore, the base case is trivial since by definition $Q_{0,1}=-\underline{e}_{1}$. The identity in 55 is another way of stating 22 . This completes our proof. 
This proposition has an avalanche of consequences. In particular, it plays a crucial role in justifying the definition of the operators $Q_{k m, k n}$. The problem, as we mentioned in the introduction, is that in this case, there are $k$ distinct points that are closest to the diagonal $(0,0) \rightarrow(k m, k n)$. inside the $k m \times k n$ lattice rectangle. Correspondingly, if $\operatorname{Split}(m, n)=(a, b)+(c, d)$, we have the following $k$ ways to split the vector $(0,0) \rightarrow(k m, k n)$ :

$$
((u-1) m+a,(u-1) n+b)+((k-u) m+c,(k-u) n+d) \quad(\text { for } 1 \leq u \leq k) .
$$

Theorems 41 and 42 allow us to overcome this problem and at the same time prove an important property of the $Q_{k m, k n}$ operators.

\section{Theorem 2.5} $u \leq k$

If $\operatorname{Split}(m, n)=(a, b)+(c, d)$ then we may set for $k>1$ and any $1 \leq$

$$
Q_{k m, k n}=\frac{1}{M}\left[Q_{(k-u) m+c,(k-u) n+d}, Q_{(u-1) m+a,(u-1) n+b}\right]
$$

Moreover, letting $\Xi=\left[\begin{array}{ll}a & c \\ b & d\end{array}\right] 2$ we also have

$$
\text { a) } \quad Q_{k, k}=\frac{q t}{1-q t} \nabla \underline{h}_{k}\left[X\left(\frac{1}{q t}-1\right)\right] \nabla^{-1} \quad \text { and } \quad \text { b) } Q_{k m, k n}=\Xi Q_{k, k}
$$

In particular it follows that for any fixed $(m, n)$ the operators $\left\{Q_{k m, k n}\right\}_{k \geq 1}$ form a commuting family.

\section{Proof}

Note first that for $(m, n)=(1,1)$ we have $\operatorname{Split}(1,1)=(1,0)+(0,1)$. Thus the right hand side of 56 becomes for any $1 \leq u \leq k$

$$
\frac{1}{M}\left[Q_{k-u, k-u+1}, Q_{u, u-1}\right]=\frac{q t}{1-q t} \nabla \underline{h}_{k}\left[X\left(\frac{1}{q t}-1\right)\right] \nabla^{-1}
$$

where the last equality is another way of writing 55. We thus immediately see that all these assertions are valid for the co-prime pair $(1,1)$, including 57 a). To deal with the case of a general co-prime pair $(m, n)$ we notice that a simple calculation gives

$$
\Xi\left[\begin{array}{c}
k-u \\
k-u+1
\end{array}\right]=\left[\begin{array}{c}
m(k-u)+c \\
n(k-u)+d
\end{array}\right], \quad \Xi\left[\begin{array}{c}
u \\
u-1
\end{array}\right]=\left[\begin{array}{c}
m(u-1)+a \\
n(u-1)+b
\end{array}\right] .
$$

\footnotetext{
${ }^{2}$ Notice $\Xi \in S L_{2}[Z]$ since $(3)$ of 24 gives $a d-b c=1$.
} 
Thus $\Xi$ maps the operators occurring on the left hand side of 58 onto the operators occurring on the right hand side of 56. Since all these operators are indexed by co-prime pairs, all the relations they satisfy are preserved by the action of the group $G$. In particular the matrix $\Xi$ will map all the equalities resulting from 58 into the desired equalities of the right hand sides of 56 . Thus 56 well defines the operator $Q_{k m, k n}$ and $57 \mathrm{~b}$ ) necessarily follows. The asserted commutativity follows just as well, since 57 a) shows that the operators $\left\{Q_{k, k}\right\}_{k \geq 1}$ form a commuting family, and the identities expressing these commutativities are preserved by $G$. This completes our proof.

An immediate corollary of Theorem 2.5 is a recursive construction of the action of the operators $Q_{k m, k n}$ on a symmetric function $F$.

\section{Algorithm}

Given a pair $(k m, k n)$ with $(m, n)$ co-prime and $k \geq 1$ :

If $k m=1$ then output $=D_{n} F$,

else

Step 1: Pick the first $1 \leq a \leq m$ such that $1=n a-m b$ where $b=$ $\lceil n a / m\rceil-1$.

Step 2: Set $(c, d)=(k m, k n)-(a, b)$.

Step 3: output $=\left(Q_{c, d} Q_{a, b} F-Q_{a, b} Q_{c, d} F\right) / M$.

Since all these operators lie in the algebra generated by the $D_{k}$, it follows from 23 that their action on a symmetric polynomial may also be given a completely explicit constant term formula.

More precisely, given any pair $(k m, k n)$ we can construct a Laurent polynomial $\Pi_{k m, k n}\left[z_{1}, \ldots, z_{k m}\right]$ such that for every symmetric polynomial $F[X]$ we have

$$
\begin{aligned}
Q_{k m, k n} F[X]=F\left[X+\sum_{i=1}^{k m} \frac{M}{z_{i}}\right] & \prod_{i=1}^{k m} \Omega\left[-z_{i} X\right] \Pi_{k m, k n}\left[z_{1}, \ldots, z_{k m}\right] \\
\times & \left.\prod_{1 \leq i<j \leq k m} \Omega\left[-M \frac{z_{i}}{z_{j}}\right]\right|_{z_{1}^{0} z_{2}^{0} \cdots z_{k m}^{0}} .
\end{aligned}
$$

In fact, the above algorithm naturally leads to the following result.

\section{Proposition 2.1}

A family of Laurent polynomials $\Pi_{k m, k n}$ that may be used in 59 can be recursively constructed as follows:

Given a pair $(k m, k n)$ with $(m, n)$ co-prime and $k \geq 1$ :

If $k m=1$ then set $\Pi_{1, n}=\frac{1}{z_{1}^{n}}$, 
else

Step 1: Pick the first $1 \leq a \leq m$ such that $1=n a-m b$ where $b=$ $\lceil n a / m\rceil-1$.

Step 2: let $(c, d)=(k m, k n)-(a, b)$.

Step 3: and set

$$
\Pi_{k m, k n}\left[Z_{1, k m}\right]=\frac{1}{M}\left(\Pi_{a, b}\left[Z_{1, a}\right] \Pi_{c, d}\left[Z_{a+1, a+c}\right]-\Pi_{c, d}\left[Z_{1, c}\right] \Pi_{c, d}\left[Z_{c+1, c+a}\right]\right) .
$$

where for convenience we have set $Z_{r, s}=\left\{z_{r}, z_{r+1}, \ldots, z_{s}\right\}$.

\section{Proof}

It suffices to show how two such operators compose after they successively act on a symmetric function. To this end suppose that

a) $\quad \mathbf{V}_{A} F[X]=\left.F\left[X+\sum_{i=1}^{a} \frac{M}{z_{i}}\right] \prod_{i=1}^{a} \Omega\left[-z_{i} X\right] \Pi_{A}\left[Z_{1, a}\right] \prod_{1 \leq i<j \leq a} \Omega\left[-M \frac{z_{i}}{z_{j}}\right]\right|_{z_{1}^{0} z_{2}^{0} \cdots z_{a}^{0}}$

c) $\quad \mathbf{V}_{C} F[X]=\left.F\left[X+\sum_{i=1}^{c} \frac{M}{z_{i}}\right] \prod_{i=1}^{c} \Omega\left[-z_{i} X\right] \Pi_{C}\left[Z_{1, c}\right] \prod_{1 \leq i<j \leq c} \Omega\left[-M \frac{z_{i}}{z_{j}}\right]\right|_{z_{1}^{0} z_{2}^{0} \cdots z_{c}^{0}}$

Applying $\mathbf{V}_{C}$ to both sides of 60 a) and using 60 c) we may write

$$
\begin{gathered}
\mathbf{V}_{C} \mathbf{V}_{A} F[X]=F\left[X+\sum_{i=1}^{a} \frac{M}{z_{i}}+\sum_{i=1}^{c} \frac{M}{z_{a+i}}\right] \prod_{i=1}^{a} \Omega\left[-z_{i}\left(X+\sum_{i=1}^{c} \frac{M}{z_{a+i}}\right)\right] \\
\times \Pi_{A}\left[Z_{1, a}\right] \Pi_{C}\left[Z_{a+1, a+c}\right] \prod_{1 \leq i<j \leq a} \Omega\left[-M \frac{z_{i}}{z_{j}}\right] \\
\times\left.\prod_{i=a+1}^{a+c} \Omega\left[-z_{i} X\right] \prod_{a+1 \leq i<j \leq a+c} \Omega\left[-M \frac{z_{i}}{z_{j}}\right]\right|_{z_{1}^{0} z_{2}^{0} \cdots z_{a+c}^{0}} \\
=F\left[X+\sum_{i=1}^{a+c} \frac{M}{z_{i}}\right] \prod_{i=1}^{a+c} \Omega\left[-z_{i} X\right] \Pi_{A}\left[Z_{1, a}\right] \Pi_{C}\left[Z_{a+1, a+c}\right] \\
\times\left.\prod_{1 \leq i<j \leq a+c} \Omega\left[-M \frac{z_{i}}{z_{j}}\right]\right|_{z_{1}^{0} z_{2}^{0} \cdots z_{a+c}^{0}}
\end{gathered}
$$

which shows that the Laurent polynomial for $\mathbf{V}_{C} \mathbf{V}_{A}$ may be taken to be $\Pi_{A}\left[Z_{1, a}\right] \Pi_{C}\left[Z_{a+1, a+c}\right]$.

It is clear, because of the multiplicity of choices of splitting a vector $(0,0) \rightarrow(k m, k n)$, that the Laurent polynomial needed in 59 is not unique. 
However, this non uniqueness goes deeper than it may be suspected, as the following identity discovered by Negut shows.

Theorem 2.6 [17]

For any co-prime pair $(m, n)$ and symmetric function $F[X]$ we have

$$
\begin{aligned}
Q_{m, n} F[X]=F[X & \left.+\sum_{i=1}^{m} \frac{M}{z_{i}}\right] \prod_{i=1}^{m} \Omega\left[-z_{i} X\right] \prod_{i=1}^{m} \frac{1}{z_{i}^{e_{i}(m, n)}} \\
& \times\left.\prod_{i=1}^{m-1} \frac{1}{\left(1-q t z_{i} / z_{i+1}\right)} \prod_{1 \leq i<j \leq m} \Omega\left[-\frac{z_{i}}{z_{j}} M\right]\right|_{z_{1}^{0} z_{2}^{0} \cdots z_{m}^{0}}
\end{aligned}
$$

where for convenience we have set

$$
e_{i}(m, n)=\left\lfloor i \frac{n}{m}\right\rfloor-\left\lfloor(i-1) \frac{n}{m}\right\rfloor .
$$

Later in this writing we will present our progress towards providing an elementary proof of this remarkable identity. Here it is most appropriate to present some of the consequences of our experimentation with the right hand side of 61 .

The first surprise is that 61 is false if $(m, n)$ is replaced by a non co-prime pair. This given, it is best to set for any pair of positive integers $(u, v)$ and symmetric function $F[X]$

$$
\begin{aligned}
\mathbf{N}_{u, v} F[X]=F[X & \left.+\sum_{i=1}^{u} \frac{M}{z_{i}}\right] \prod_{i=1}^{u} \Omega\left[-z_{i} X\right] \prod_{i=1}^{u} \frac{1}{z_{i}^{e_{i}(u, v)}} \\
& \times\left.\prod_{i=1}^{u-1} \frac{1}{\left(1-q t z_{i} / z_{i+1}\right)} \prod_{1 \leq i<j \leq u} \Omega\left[-\frac{z_{i}}{z_{j}} M\right]\right|_{z_{1}^{0} z_{2}^{0} \ldots z_{u}^{0}}
\end{aligned}
$$

and refer to it as the Negut operator.

The next surprise is that computer experimentation led us to formulate the following remarkable

\section{Conjecture 2.1}

For all $k \geq 1$ and all $F[X]$ we have

$$
\mathbf{N}_{k, k} F[X]=\nabla \underline{e}_{k} \nabla^{-1} F[X] .
$$

This given, the relation of Negut's $\mathbf{N}_{k, k}$ operator to the $Q_{k m, k n}$ operators should be given by the following identity. 


\section{Theorem 2.6}

$$
\mathbf{N}_{k, k}=(-1)^{k} \sum_{\lambda \vdash k} m_{\lambda}\left[\frac{q t}{q t-1}\right]\left(\frac{1-q t}{q t}\right)^{l(\lambda)} \prod_{i=1}^{l(\lambda)} Q_{\lambda_{i}, \lambda_{i}}
$$

\section{Proof}

Note first that we may write for any two expressions $A, B$

$$
h_{k}[A B]=\sum_{\lambda \vdash k} m_{\lambda}[B] h_{\lambda}[A] .
$$

Letting $A=X\left(\frac{1}{q t}-1\right)$ and $B=\frac{q t}{q t-1}$ gives

$$
(-1)^{k} e_{k}[X]=h_{k}[-X]=\sum_{\lambda \vdash k} m_{\lambda}\left[\frac{q t}{q t-1}\right] h_{\lambda}\left[X\left(\frac{1}{q t}-1\right)\right] .
$$

Thus conjugating both sides by $\nabla$ gives

$$
\nabla \underline{e}_{k} \nabla^{-1}=(-1)^{k} \sum_{\lambda \vdash k} m_{\lambda}\left[\frac{q t}{q t-1}\right] \nabla \underline{h}_{\lambda}\left[X\left(\frac{1}{q t}-1\right)\right] \nabla^{-1} .
$$

But using 57 a) we easily derive that

$$
\nabla \underline{h}_{\lambda}\left[X\left(\frac{1}{q t}-1\right)\right] \nabla^{-1}=\left(\frac{1-q t}{q t}\right)^{l(\lambda)} \prod_{i=1}^{l(\lambda)} Q_{\lambda_{i}, \lambda_{i}}
$$

and we see that, given 64 , the identity in 65 is simply another way of writing 66.

Thus it would follow from Conjecture 2.1 that the operators $\mathbf{N}_{k, k}$ are in the algebra generated by the $D_{k}$ operators. This fact plus a variety of reasons, including experimental evidence, suggested that for the matrix $\Xi$ of Theorem 2.5 we should have $\Xi \mathbf{N}_{k, k}=\mathbf{N}_{k m, k n}$. This given, applying $\Xi$ to both sides of 65 yields the following extension of Conjecture 2.1.

\section{Conjecture 2.2}

For all co-prime $(m, n)$ and $k \geq 1$ we have

$$
\mathbf{N}_{k m, k n}=(-1)^{k} \sum_{\lambda \vdash k} m_{\lambda}\left[\frac{q t}{q t-1}\right]\left(\frac{1-q t}{q t}\right)^{l(\lambda)} \prod_{i=1}^{l(\lambda)} Q_{\lambda_{i} m, \lambda_{i} n} .
$$

The same sequence of steps carried out in the construction of the operator in the right hand side of 67 , can be used to create an infinite family of operators in the algebra generated by the $D_{k}$ operators. In fact we need 
only replace $e_{k}$ by any symmetric function of the same degree in the manipulations carried out in the proof of Theorem 2.6.

To carry this out it is convenient to set for any partition $\lambda=\left(\lambda_{1}, \lambda_{2}, \cdots\right.$, $\left.\lambda_{l}\right)$

$$
h_{\lambda}[X ; q, t]=\left(\frac{q t}{1-q t}\right)^{l} \prod_{i=1}^{l} h_{\lambda_{i}}[X(1 / q t-1)]
$$

and notice that the collection $\left\{h_{\lambda}[X ; q, t]\right\}_{\lambda}$ is a symmetric function basis.

This given, we proceed as follows:

\section{Definition 2.1}

Given any symmetric function $G$ that is homogeneous of degree $k$ and any co-prime pair $(m, n)$ :

Step 1: Construct the expansion

$$
G=\sum_{\lambda \vdash k} c_{\lambda}(q, t) h_{\lambda}[X ; q, t] .
$$

Step 2: Set

$$
\mathbf{G}_{k m, k n}=\sum_{\lambda \vdash k} c_{\lambda}(q, t) \prod_{i=1}^{l(\lambda)} Q_{m \lambda_{i}, n \lambda_{i}} .
$$

\section{Remark 2.1}

It is easily seen that the operator on the right hand side of 67 is simply $\mathbf{G}_{k m, k n}$ for $G=e_{k}$. This immediately gives rise to a variety of questions. To begin, are there ways to modify the definition of the Negut operator $\mathbf{N}_{u, v}$ to obtain the action of $\mathbf{G}_{k m, k n}$ for some other choices of $G$. Secondly, we have $\mathbf{G}_{k, k}=\nabla G \nabla^{-1}$ whenever $G$ is of degree $k$. Hence the well-known fact that $\nabla e_{k}$ is Schur positive combined with the fact that $\mathbf{N}_{k, k}=\nabla e_{k} \nabla^{-1}$ makes us wonder what cases of Schur positivity may occur for other choices of $G$. Of course it is experimentally well known that $\pm \nabla s_{\lambda}$, with an appropriate choice of the sign, is Schur positive. We may then ask what bi-graded $S_{n}$ modules may have Frobenius characteristics given by the symmetric polynomials resulting from actions of the operators $\mathbf{G}_{k m, k n}$.

It is also conjectured by Haglund et al. [13] that a refinement of the polynomial $\nabla e_{k}$ may also be obtained as an appropriate enumerator of parking functions. Using this conjecture Y. Kim in a recent thesis [15] shows that for an infinite variety of 2-row and 2-column partitions the polynomial $\pm \nabla s_{\lambda}$ should also be obtained as an enumerator of parking functions. Can other choices of $G$ lead to similar findings? It develops that these questions have some truly surprising answers. The reader is referred to a forthcoming arti- 
cle [3] where the $\mathbf{G}_{k m, k n}$ operators, for a variety of choices of the symmetric function $G$ are shown to be closely connected to the combinatorics of the rational "Parking Functions" constructed by Hikita in [14].

\section{The Negut operators and the SSS trick}

Throughout this section, we will use the notations $S_{k}$ and $S y m_{k}$ for the symmetric group of order $k$ and symmetrization with respect to $Z_{k}=$ $\left\{z_{1}, \ldots, z_{k}\right\}$ respectively. It will also be useful to write $Z_{a, b}, S_{a, b}$ and $S y m_{a, b}$ when restricting to the variables $\left\{z_{a}, z_{a+1}, \ldots z_{b}\right\}$ for some $a \leq b$.

The problem we deal with in this section is best understood if we start with an example. Suppose we want to program on the computer the action of the operator $Q_{5,3}$. Now using 59 for $k=1$ and $(m, n)=(5,3)$ we get (70)

$Q_{5,3} F[X]=\left.F\left[X+\sum_{i=1}^{5} \frac{M}{z_{i}}\right] \prod_{i=1}^{5} \Omega\left[-z_{i} X\right] \Pi_{5,3}\left[z_{1}, z_{2}, \ldots, z_{5}\right] \prod_{1 \leq i<j \leq 5} \Omega\left[-M \frac{z_{i}}{z_{j}}\right]\right|_{z_{1}^{0} z_{2}^{0} \cdots z_{5}^{0}}$

where the Laurent polynomial $\Pi_{5,3}\left[z_{1}, z_{2}, \ldots, z_{5}\right]$ may be obtained by the recursion in Proposition 2.1. In this case it is simpler to construct it directly from the binary tree below. By associating the $m \times n$ grid with the operator $Q_{m, n}$, the successive splitting depicted by this tree immediately gives

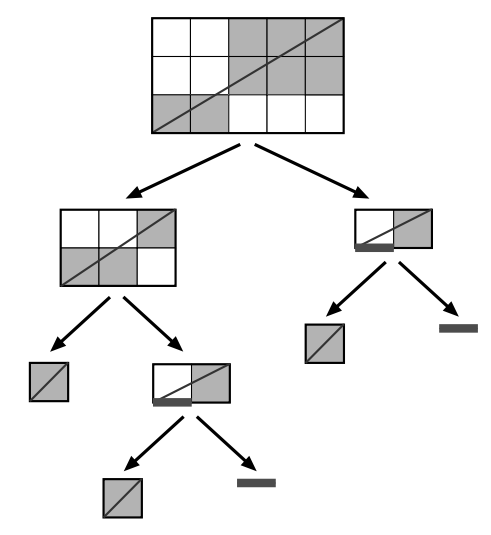

$$
\begin{aligned}
Q_{5,3}=\frac{1}{M}[ & {\left.\left[D_{1},\left[D_{1}, D_{0}\right]\right],\left[D_{1}, D_{0}\right]\right] } \\
=\frac{1}{M}( & \left(D_{1}\left(D_{1} D_{0}-D_{0} D_{1}\right)-\left(D_{1} D_{0}-D_{0} D_{1}\right) D_{1}\right)\left(D_{1} D_{0}-D_{0} D_{1}\right)- \\
& \left.\quad-\left(D_{1} D_{0}-D_{0} D_{1}\right)\left(D_{1}\left(D_{1} D_{0}-D_{0} D_{1}\right)-\left(D_{1} D_{0}-D_{0} D_{1}\right) D_{1}\right)\right)
\end{aligned}
$$

Expanding this out we get 


$$
\begin{aligned}
& Q_{5,3}=\frac{1}{M^{4}}\left(D_{1} D_{1} D_{0} D_{1} D_{0}-3 D_{1} D_{0} D_{1} D_{1} D_{0}+2 D_{0} D_{1} D_{1} D_{1} D_{0}\right. \\
&-D_{1} D_{1} D_{0} D_{0} D_{1}+ 4 D_{1} D_{0} D_{1} D_{0} D_{1}-3 D_{0} D_{1} D_{1} D_{0} D_{1} \\
&\left.-D_{1} D_{0} D_{0} D_{1} D_{1}+D_{0} D_{1} D_{0} D_{1} D_{1}\right)
\end{aligned}
$$

from which we derive that

$$
\begin{aligned}
\Pi_{5,3}\left(z_{1}, z_{2}, \ldots, z_{5}\right)=\frac{1}{M^{4}}\left(\frac{1}{z_{2} z_{4} z_{5}}\right. & -3 \frac{1}{z_{2} z_{3} z_{5}}+2 \frac{1}{z_{2} z_{3} z_{4}}-\frac{1}{z_{1} z_{4} z_{5}} \\
& \left.+41) \quad \frac{1}{z_{1} z_{3} z_{5}}-3 \frac{1}{z_{1} z_{3} z_{4}}-\frac{1}{z_{1} z_{2} z_{5}}+\frac{1}{z_{1} z_{2} z_{4}}\right) .
\end{aligned}
$$

Now by 63 for $u, v=5,3$, we derive that Negut's result is

$$
Q_{5,3} F[X]=\mathbf{N}_{5,3} F[X] \quad \text { (for all symmetric functions } F[X] \text { ) }
$$

with

$$
\begin{aligned}
\mathbf{N}_{5,3} F[X]=F[X & \left.+\sum_{i=1}^{5} \frac{M}{z_{i}}\right] \prod_{i=1}^{5} \Omega\left[-z_{i} X\right] \prod_{i=1}^{5} \frac{1}{z_{i}^{e_{i}(m, n)}} \\
& \times\left.\prod_{i=1}^{4} \frac{1}{\left(1-q t z_{i} / z_{i+1}\right)} \prod_{1 \leq i<j \leq 5} \Omega\left[-\frac{z_{i}}{z_{j}} M\right]\right|_{z_{1}^{0} z_{2}^{0} \cdots z_{5}^{0}}
\end{aligned}
$$

To gauge the simplicity of this formula we need only compute the mono$\operatorname{mial} \prod_{i=1}^{5} \frac{1}{z_{i}^{e_{i}(m, n)}}$.

Note that the definition in 62 , giving

$$
e_{i}(m, n)=\left\lfloor i \frac{n}{m}\right\rfloor-\left\lfloor(i-1) \frac{n}{m}\right\rfloor,
$$

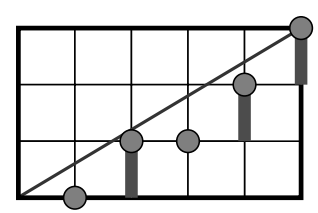

geometrically simply means finding, for each $i$, the highest lattice point $\left(i, f_{i}\right)$ on the line $x=i$ that is below the main diagonal of the lattice rectangle $m \times n$, then setting $e_{i}=f_{i}-f_{i-1}$. Thus the adjacent display shows that the monomial in 72 is simply $z_{2} z_{4} z_{5}$.

Now from Theorem 2.1 we derive that 72 can hold true if and only if (74)

$$
\operatorname{Sym}_{5}\left(\left(\Pi_{5,3}\left(z_{1}, \ldots, z_{5}\right)-z_{2} z_{4} z_{5} \prod_{i=1}^{4} \frac{1}{\left(1-q t \frac{z_{i}}{z_{i+1}}\right)}\right) \prod_{1 \leq i<j \leq 5} \Omega\left[-\frac{z_{i}}{z_{j}} M\right]\right)=0,
$$

an identity that should be verifiable by computer.

From this example it is easy to deduce the following general result. 


\section{Theorem 3.1}

Let $m, n>0$ be coprime. The equality

$$
Q_{m, n} F[X]=\mathbf{N}_{m, n} F[X]
$$

holds true for all symmetric functions $F[X]$ if and only if

$$
\begin{gathered}
\operatorname{Sym}_{m}\left(\left(\prod_{m, n}\left(z_{1}, z_{2}, \ldots, z_{m}\right)-\prod_{i=1}^{m} \frac{1}{z_{i}^{\left\lfloor i \frac{n}{m}\right\rfloor-\left\lfloor(i-1) \frac{n}{m}\right\rfloor}} \prod_{i=1}^{m-1} \frac{1}{\left(1-q t \frac{z_{i}}{z_{i+1}}\right)}\right)\right. \\
\left.\times \prod_{1 \leq i<j \leq m} \Omega\left[-\frac{z_{i}}{z_{j}} M\right]\right)=0 .
\end{gathered}
$$

The identity in 73 actually was not entirely verifiable on a laptop computer. The problem is not carrying out the symmetrization, but recognizing that the result of symmetrization is actually equal to zero. By setting $z_{i}=\theta^{i}$ in 74 then MAPLE is able to recognize that the resulting expression simplifies to zero. On the other hand, for the examples in which $m \leq 4$ such as those depicted below
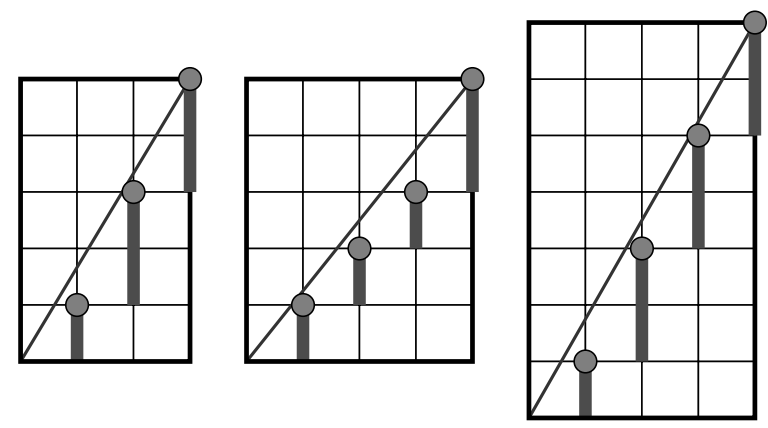

the Negut equality $Q_{m, n}=\mathbf{N}_{m, n}$ can be verified even on a laptop in a few seconds. Moreover, as long as $m \leq 4$, we can easily obtain a computer proof of 76. More precisely

\section{Theorem 3.2}

For all co-prime pairs $(m, n)$ with $m \leq 4$ we have

$$
Q_{m, n}=\mathbf{N}_{m, n}
$$

\section{Proof}

We might suspect that $Q_{m, n}-\mathbf{N}_{m, n}=0$ should imply that $Q_{m, n+m}-$ $\mathbf{N}_{m, n+m}=0$ by the action of the matrix $S=\left[\begin{array}{ll}1 & 0 \\ 1 & 1\end{array}\right]$. Of course we know that 
$S Q_{m, n}=Q_{m, n+m}$, however at this moment, we have no way to justify acting by $S$ on $\mathbf{N}_{m, n}$. Nevertheless, the idea can be salvaged, for more elementary reason. Simply observe that it follows from 42 that

$$
\Pi_{m, n+m}\left(z_{1}, z_{2}, \ldots, z_{m}\right)=\frac{\Pi_{m, n}\left(z_{1}, z_{2}, \ldots, z_{m}\right)}{z_{1} z_{2} \cdots z_{m}} .
$$

At the same we can also see that

$$
\prod_{i=1}^{m} \frac{1}{z_{i}^{\left\lfloor\frac{i n+m}{m}\right\rfloor}-\left\lfloor(i-1) \frac{n+m}{m}\right\rfloor}=\frac{1}{z_{1} z_{2} \cdots z_{m}} \prod_{i=1}^{m} \frac{1}{z_{i}^{\left\lfloor\frac{n}{m}\right\rfloor-\left\lfloor(i-1) \frac{n}{m}\right\rfloor}}
$$

and we can immediately conclude that the validity of 76 for a co-prime pair $(m, n)$ forces the validity of 76 for $(m, n+k m)$ for any $k \geq 1$. Thus to prove 77 for all pairs $(2,1+2 k),(3,1+3 k),(3,2+3 k),(4,1+4 k),(4,3+4 k)$ it is sufficient to check it by computer for $k=0$. This can be readily obtained in MAPLE or MATHEMATICA. It is conceivable that by clever means we could succeed in pushing the above computer proof to $m=5$, but beyond that point it is better to proceed by a more powerful theoretical approach.

To this end, a moment's reflection should make us plainly see how the "shuffle algebra" arises within the present context. In fact, suppose we define as the "product" of two symmetric functions $F\left[Z_{a}\right], G\left[Z_{b}\right]$ as the symmetric function $(F \otimes G)\left[Z_{a+b}\right]$ defined by setting

$$
F\left[Z_{a}\right] \otimes G\left[Z_{b}\right]=\operatorname{Sym}_{a+b}\left(F\left[Z_{a}\right] G\left[Z_{a+1, a+b}\right] \Omega\left[-M Z_{a} Z_{a+1, a+b}^{-1}\right]\right)
$$

where for an alphabet $Z$ the symbol " $Z^{-1}$ " denotes the sum of the inverses of its letters. Note that 79 can also be rewritten as

$$
\left.F\left[Z_{a}\right] \otimes G\left[Z_{b}\right]=\frac{a ! b !}{(a+b) !} \sum_{\substack{A+B=[a+b] \\|A|=a,|B|=b}} F\left[Z_{A}\right] G\left[Z_{B}\right] \Omega\left[-M Z_{A} Z_{B}^{-1}\right]\right)
$$

This given, let us set

$$
\mathbf{U}_{m, n}\left[Z_{m}\right]=\operatorname{Sym}_{m}\left(\Pi_{m, n}\left[Z_{m}\right] \prod_{1 \leq i<j \leq m} \Omega\left[-M z_{i} / z_{j}\right]\right) .
$$

Then from Proposition 2.1 and 79 it follows that.

\section{Proposition 3.1}

For all co-prime pairs $(m, n)$ with $m>n$ we have 


$$
\mathbf{U}_{m, n}\left[Z_{m}\right]=\frac{1}{M}\left(\mathbf{U}_{c, d}\left[Z_{c}\right] \otimes \mathbf{U}_{a, b}\left[Z_{a}\right]-\mathbf{U}_{a, b}\left[Z_{a}\right] \otimes \mathbf{U}_{c, d}\left[Z_{c}\right]\right)
$$

\section{Proof}

Note first that

$$
\begin{gathered}
\operatorname{Sym}_{a+c}\left(\Pi_{c, d}\left[Z_{c}\right] \Pi_{a, b}\left[Z_{c+1, c+a}\right] \prod_{1 \leq i<j \leq a+b} \Omega\left[-M z_{i} / z_{j}\right]\right)= \\
=\frac{c ! a !}{(c+a) !} \sum_{\tau} \tau\left(\operatorname{Sym}_{c}\left(\Pi_{c, d}\left[Z_{c}\right] \prod_{1 \leq i<j \leq c} \Omega\left[-M z_{i} / z_{j}\right]\right)\right. \\
\times S y m_{c+1, c+a}\left(\prod_{a, b}\left[Z_{c+1, c+a}\right] \prod_{c+1 \leq i<j \leq c+a} \Omega\left[-M z_{i} / z_{j}\right]\right) \\
\left.\times \prod_{\substack{1 \leq i \leq c \\
c+1 \leq j \leq c+a}} \Omega\left[-M z_{i} / z_{j}\right]\right) \\
=\frac{c ! a !}{(c+a) !} \sum_{\tau}\left(\mathbf{U}_{c, d}\left[Z_{c}\right] \mathbf{U}_{a, b}\left[Z_{c+1, c+a}\right] \prod_{\substack{1 \leq i \leq c \\
c+1 \leq j \leq c+a}} \Omega\left[-M z_{i} / z_{j}\right]\right)
\end{gathered}
$$

(by 80$)=\mathbf{U}_{c, d}\left[Z_{c}\right] \otimes \mathbf{U}_{a, b}\left[Z_{a}\right]$

where the sum is over the left coset representatives $\tau$ of the subgroup $S_{c} \times$ $S_{c+1, c+a} \subseteq S_{a+c}$. It should now be quite clear that the second term in 82 can be obtained in an entirely analogous manner. Thus to complete our argument, we need only to use the recursion

$$
\Pi_{m, n}\left[Z_{m}\right]=\frac{1}{M}\left(\Pi_{c, d}\left[Z_{c}\right] \Pi_{a, b}\left[Z_{c+1, c+a}\right]-\Pi_{a, b}\left[Z_{a}\right] \Pi_{c, d}\left[Z_{a+1, a+c}\right]\right) .
$$

This suggests an inductive approach to the proof of the equality $Q_{m, n}=$ $\mathbf{N}_{m, n}$. Start by showing that if $\operatorname{Split}(m, n)=(a, b)+(c, d)$ then

$$
\mathbf{N}_{m, n}=\frac{1}{M}\left[\mathbf{N}_{c, d}, \mathbf{N}_{a, b}\right]
$$

For convenience let us set

$$
\mathbf{V}_{m, n}\left[Z_{m}\right]=\operatorname{Sym}_{m}\left(\Xi_{m, n}\left[Z_{m}\right] \prod_{1 \leq i<j \leq m} \Omega\left[-M z_{i} / z_{j}\right]\right)
$$

with 


$$
\begin{aligned}
\Xi_{m, n}\left[Z_{m}\right] & =\prod_{i=1}^{m} \frac{1}{z_{i}^{\left\lfloor\frac{n}{m}\right\rfloor}-\left\lfloor(i-1) \frac{n}{m}\right\rfloor} \prod_{i=1}^{m-1} \frac{1}{1-q t z_{i} / z_{i+1}} \\
& =\frac{1}{z_{m}^{n}} \prod_{i=1}^{m-1} \frac{\left(z_{i} / z_{i+1}\right)^{-\left\lfloor i \frac{n}{m}\right\rfloor}}{1-q t z_{i} / z_{i+1}} .
\end{aligned}
$$

Note that 63 for $(u, v)=(m, n)$ may be written as

$$
\begin{array}{r}
\mathbf{N}_{m, n} F[X]=F\left[X+\sum_{i=1}^{m} \frac{M}{z_{i}}\right] \prod_{i=1}^{m} \Omega\left[-z_{i} X\right] \\
\left.\quad \Xi_{m, n}\left[Z_{m}\right] \prod_{1 \leq i<j \leq m} \Omega\left[-\frac{z_{i}}{z_{j}} M\right]\right|_{z_{1}^{0} z_{2}^{0} \cdots z_{m}^{0}}
\end{array}
$$

and since $\Xi_{1, n}\left(Z_{1}\right)=\frac{1}{z_{1}}$ it follows that $\mathbf{N}_{1, n}=D_{1}=Q_{1, n}$ for all $n \geq 1$. Thus a proof of 84 is all that is needed to prove the Negut equality $Q_{m, n}=\mathbf{N}_{m, n}$.

As further evidence of the isomorphism between the shuffle algebra and the algebra generated by the $D_{k}$ operators, we must point out that from Theorems 39 and 87 we may easily derive the following.

\section{Proposition 3.2}

For a co-prime pair $(m, n)$ with $\operatorname{Split}(m, n)=(a, b)+(c, d)$ we have

$$
\begin{aligned}
\mathbf{N}_{m, n} & =\frac{1}{M}\left[\mathbf{N}_{c, d}, \mathbf{N}_{a, b}\right] \Longleftrightarrow \\
\mathbf{V}_{m, n}\left[Z_{m}\right] & =\frac{1}{M}\left(\mathbf{V}_{c, d}\left[Z_{c}\right] \otimes \mathbf{V}_{a, b}\left[Z_{a}\right]-\mathbf{V}_{a, b}\left[Z_{a}\right] \otimes \mathbf{V}_{c, d}\left[Z_{c}\right]\right) .
\end{aligned}
$$

The following general result enabled us to obtain a computer proof of Negut's equality 75 for all $m \leq 7$.

\section{Theorem 3.3}

If $m>n$ is a co-prime pair with $\operatorname{Split}(m, n)=(a, b)+(c, d)$ then the identity

$$
\mathbf{N}_{m, n}=\frac{1}{M}\left[\mathbf{N}_{c, d}, \mathbf{N}_{a, b}\right]
$$

holds true if and only if

$$
\begin{aligned}
\operatorname{Sym}_{m}\left(\frac{\Xi_{m, n}\left[Z_{m}\right]}{z_{a+1} z_{c}}\left(z_{a+1} z_{c+1}-t z_{a+1} z_{c}-q z_{a+1} z_{c}+q t z_{a} z_{c}\right)\right. \\
\left.\times \prod_{1 \leq i<j \leq m} \Omega\left[-\frac{z_{i}}{z_{j}} M\right]\right)=0 .
\end{aligned}
$$


In particular the validity of 89 forces the equality

$$
\mathbf{N}_{m, n}=Q_{m, n} .
$$

\section{Proof}

From 87 and Theorem 2.1 we derive that 88 holds true if and only if

$$
\begin{gathered}
\operatorname{Sym}_{m}\left(\left(M \Xi_{m, n}\left[Z_{m}\right]-\left(\Xi_{a, b}\left[Z_{a}\right] \Xi_{c, d}\left[Z_{a+1, a+c}\right]-\Xi_{c, d}\left[Z_{c}\right] \Xi_{a, b}\left[Z_{c+1, c+a}\right]\right)\right)\right. \\
\left.\times \prod_{1 \leq i<j \leq m} \Omega\left[-\frac{z_{i}}{z_{j}} M\right]\right)=0 .
\end{gathered}
$$

To compute the first factor within $S y m_{m}$ we need to consider the two cases $a<c$ and $a>c$ which are schematically depicted in the display below.
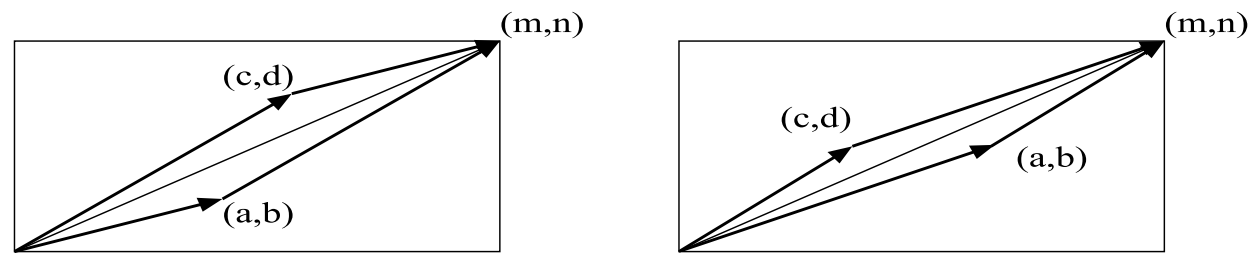

Note first that in each case there are no lattice points within the parallelogram formed by the arrows. Thus the set of highest lattice points $\left(i, f_{i}\right)$ below the diagonal $(0,0) \rightarrow(m, n)$ is the same as the set of highest lattice points $\left(i, f_{i}\right)$ below the vector sum $(a, b)+(c, d)$, except for $(a, b)$. This gives

$$
\begin{aligned}
& \left\lfloor i \frac{n}{m}\right\rfloor=\left\lfloor i \frac{b}{a}\right\rfloor \quad(\text { for } 1 \leq i \leq a) \quad \text { and } \\
& \left\lfloor i \frac{n}{m}\right\rfloor=b+\left\lfloor(i-a) \frac{d}{c}\right\rfloor \quad(\text { for } a+1 \leq i \leq a+c=m) .
\end{aligned}
$$

Thus 86 gives

$$
\begin{array}{r}
\Xi_{a, b}\left[Z_{a}\right] \Xi_{c, d}\left[Z_{a+1, a+c}\right]=\frac{1}{z_{a}^{b}} \prod_{i=1}^{a-1} \frac{\left(z_{i} / z_{i+1}\right)^{-\left\lfloor i \frac{b}{a}\right\rfloor}}{1-q t z_{i} / z_{i+1}} \frac{1}{z_{a+c}^{d}} \prod_{i=a+1}^{a+c-1} \frac{\left(z_{i} / z_{i+1}\right)^{-\left\lfloor(i-a) \frac{d}{c}\right\rfloor}}{1-q t z_{i} / z_{i+1}} \\
=\left(\frac{z_{a}}{z_{a+1}}\right)^{b}\left(1-q t \frac{z_{a}}{z_{a+1}}\right) \frac{1}{z_{a}^{b}} \prod_{i=1}^{a} \frac{\left(z_{i} / z_{i+1}\right)^{-\left\lfloor i \frac{n}{m}\right\rfloor}}{1-q t z_{i} / z_{i+1}} \frac{1}{z_{m}^{d}} \prod_{i=a+1}^{m-1} \frac{\left(z_{i} / z_{i+1}\right)^{b-\left\lfloor i \frac{n}{m}\right\rfloor}}{1-q t z_{i} / z_{i+1}} \\
=\frac{1}{z_{a+1}^{b}}\left(1-q t \frac{z_{a}}{z_{a+1}}\right) \frac{z_{m}^{n}}{z_{m}^{d}} \Xi_{m, n}\left[Z_{m}\right]\left(z_{a+1} / z_{m}\right)^{b}=\left(1-q t \frac{z_{a}}{z_{a+1}}\right) \Xi_{m, n}\left[Z_{m}\right] .
\end{array}
$$


For the same reason, in each case the set of highest lattice points $\left(i, f_{i}\right)$ below the diagonal $(0,0) \rightarrow(m, n)$ is the same as the set highest lattice points $\left(i, f_{i}\right)$ below the reversed vector sum $(c, d)+(a, b)$, except for $(c, d)$. This gives

$$
\begin{aligned}
& \left\lfloor i \frac{n}{m}\right\rfloor=\left\lfloor i \frac{d}{c}\right\rfloor-\chi(i=d)(\text { for } 1 \leq i \leq c) \text { and } \\
& \left\lfloor i \frac{n}{m}\right\rfloor=d+\left\lfloor(i-c) \frac{b}{a}\right\rfloor(\text { for } c+1 \leq i \leq c+a=m)
\end{aligned}
$$

thus again from 86 we get

$$
\begin{aligned}
& \Xi_{c, d}\left[Z_{c}\right] \Xi_{a, b}\left[Z_{c+1, c+a}\right]=\frac{1}{z_{c}^{d}} \prod_{i=1}^{c-1} \frac{\left(z_{i} / z_{i+1}\right)^{-\left\lfloor i \frac{d}{c}\right\rfloor}}{1-q t z_{i} / z_{i+1}} \frac{1}{z_{c+a}^{b}} \prod_{i=c+1}^{c+a-1} \frac{\left(z_{i} / z_{i+1}\right)^{-\left\lfloor(i-c) \frac{b}{a}\right\rfloor}}{1-q t z_{i} / z_{i+1}} \\
& =\left(\frac{z_{c}}{z_{c+1}}\right)^{d-1}\left(1-q t \frac{z_{c}}{z_{c+1}}\right) \frac{1}{z_{c}^{d}} \prod_{i=1}^{c} \frac{\left(z_{i} / z_{i+1}\right)^{-\left\lfloor i \frac{n}{m}\right\rfloor}}{1-q t z_{i} / z_{i+1}} \frac{1}{z_{m}^{b}} \prod_{i=c+1}^{m-1} \frac{\left(z_{i} / z_{i+1}\right)^{d-\left\lfloor i \frac{n}{m}\right\rfloor}}{1-q t z_{i} / z_{i+1}} \\
& =\frac{z_{c}^{-1}}{z_{c+1}^{d-1}}\left(1-q t \frac{z_{c}}{z_{c+1}}\right) \frac{z_{m}^{n}}{z_{m}^{b}} \Xi_{m, n}\left[Z_{m}\right]\left(z_{c+1} / z_{m}\right)^{d} \\
& =z_{c+1} z_{c}^{-1}\left(1-q t \frac{z_{c}}{z_{c+1}}\right) \Xi_{m, n}\left[Z_{m}\right] .
\end{aligned}
$$

Combining these two identities we get

$$
\begin{gathered}
\left(M \Xi_{m, n}\left[Z_{m}\right]-\left(\Xi_{a, b}\left[Z_{a}\right] \Xi_{c, d}\left[Z_{a+1, a+c}\right]-\Xi_{c, d}\left[Z_{c}\right] \Xi_{a, b}\left[Z_{c+1, c+a}\right]\right)\right)= \\
=\Xi_{m, n}\left[Z_{m}\right]\left((1-t)(1-q)-\left(1-q t \frac{z_{a}}{z_{a+1}}\right)+z_{c+1} z_{c}^{-1}\left(1-q t \frac{z_{c}}{z_{c+1}}\right)\right) \\
=\frac{\Xi_{m, n}\left[Z_{m}\right]}{z_{a+1} z_{c}}\left(z_{a+1} z_{c}(1-t-q+q t)-\left(z_{a+1} z_{c}-q t z_{a} z_{c}\right)\right. \\
\left.+z_{a+1} z_{c+1}\left(1-q t \frac{z_{c}}{z_{c+1}}\right)\right) \\
=\frac{\Xi_{m, n}\left[Z_{m}\right]}{z_{a+1} z_{c}}\left(z_{a+1} z_{c}(-t-q)+q t z_{a} z_{c}+z_{a+1} z_{c+1}\right) .
\end{gathered}
$$

This shows that 90 is equivalent to 89 and completes our proof.

To this date we have not yet been able to prove 89 in full generality. However, Theorem 3.3 has the following immediate corollary.

\section{Theorem 3.4}

For any co-prime pair $(m, n)$ we have

$$
\left.\mathbf{N}_{m, n}\right|_{t=1 / q}=\left.Q_{m, n}\right|_{t=1 / q} .
$$




\section{Proof}

It is sufficient to verify 89 for $t=1 / q$. To begin notice that

$$
\Omega[-u M]=\frac{q(1-u)^{2}}{(q-u)(1-q u)}
$$

This gives

$$
\left.\prod_{1 \leq i<j \leq m} \Omega\left[-M z_{i} / z_{j}\right]\right|_{t=1 / q}=q^{\left(\begin{array}{c}
m \\
2
\end{array}\right)} \prod_{1 \leq i<j \leq m} \frac{\left(z_{i}-z_{j}\right)^{2}}{\left(q z_{j}-z_{i}\right)\left(z_{j}-q z_{i}\right)}
$$

which is easily seen to be a symmetric rational function. Thus we only need to show that

$$
\operatorname{Sym}_{m}\left(\left.\Xi_{m, n}\left[Z_{m}\right]\right|_{t=1 / q}\left(z_{c+1} / z_{c}-(q+1 / q)+z_{a} / z_{a+1}\right)\right)=0
$$

or equivalently that

$$
\operatorname{Asym}_{m}\left(\left.\Xi_{m, n}\left[Z_{m}\right]\right|_{t=1 / q} \Delta\left[Z_{m}\right]\left(z_{c+1} / z_{c}-(q+1 / q)+z_{a} / z_{a+1}\right)\right)=0
$$

with $\Delta\left[Z_{m}\right]=\prod_{1 \leq i<j \leq m}\left(z_{j}-z_{i}\right)$ and "Asym ${ }_{m}$ " denoting $S_{m}$ antisymmetrization.

Next notice that since we need only prove 91 for $1 \leq n \leq m-1$ we can assume, here and after, that $\frac{n}{m}<1$ and we may write

$$
\begin{aligned}
\left.\Xi_{m, n}\left[Z_{m}\right]\right|_{t=1 / q} \Delta\left[Z_{m}\right] & =\prod_{i=1}^{m} \frac{1}{z_{i}^{\left\lfloor i \frac{n}{m}\right\rfloor-\left\lfloor(i-1) \frac{n}{m}\right\rfloor}} \prod_{i=1}^{m-1} \frac{z_{i+1}}{z_{i+1}-z_{i}} \Delta\left[Z_{m}\right] \\
& =\prod_{i=2}^{m} \frac{z_{i}}{z_{i}^{\left\lfloor i \frac{n}{m}\right\rfloor}-\left\lfloor(i-1) \frac{n}{m}\right\rfloor} \prod_{i=1}^{m-2} \prod_{j=i+2}^{m}\left(z_{j}-z_{i}\right) .
\end{aligned}
$$

Thus we are reduced to showing that

$$
\begin{aligned}
\operatorname{Asym}_{m}\left(\left(\frac{z_{c+1}}{z_{c}}-\right.\right. & \left.(q+1 / q)+\frac{z_{a}}{z_{a+1}}\right) \\
& \left.\times \prod_{i=2}^{m} \frac{z_{i}}{z_{i}^{\left\lfloor i \frac{n}{m}\right\rfloor-\left\lfloor(i-1) \frac{n}{m}\right\rfloor}} \prod_{i=1}^{m-2} \prod_{j=i+2}^{m}\left(z_{j}-z_{i}\right)\right)=0 .
\end{aligned}
$$


For the pairs $(m, n)$ with $1 \leq n \leq m-2$ we can prove that the expression to be anti-symmetrized is actually a homogeneous polynomial of degree less than $\left(\begin{array}{c}m \\ 2\end{array}\right)$, which is the minimum possible degree for which Asym $_{m}$ can yield something other than zero. For the pair $(m, m-1)$ we will need to prove 92 by a direct brute force argument.

Notice first that for any $1 \leq i \leq m$ we have

$$
\left\lfloor i \frac{n}{m}\right\rfloor-\left\lfloor(i-1) \frac{n}{m}\right\rfloor \leq 1 .
$$

In fact setting $\left\lfloor i \frac{n}{m}\right\rfloor=r$ and letting $\epsilon=i \frac{n}{m}-r$ we derive

$$
\left\lfloor(i-1) \frac{n}{m}\right\rfloor=\left\lfloor r+\epsilon-\frac{n}{m}\right\rfloor= \begin{cases}r+\left\lfloor\epsilon-\frac{n}{m}\right\rfloor=r & \text { if } \epsilon \geq \frac{n}{m} \\ r-\left\lceil\frac{n}{m}-\epsilon\right\rceil=r-1 & \text { if } \epsilon<\frac{n}{m} .\end{cases}
$$

This proves 93 .

Let us now suppose that $n \leq m-2$.

In view of 93 to show that the expression inside $A_{s y m_{m}}$ is a polynomial we need only show that

$$
\text { 1) } \left.\left\lfloor c \frac{n}{m}\right\rfloor-\left\lfloor(c-1) \frac{n}{m}\right\rfloor=0 \quad \text { and } \quad 2\right) \quad\left\lfloor(a+1) \frac{n}{m}\right\rfloor-\left\lfloor a \frac{n}{m}\right\rfloor=0
$$

This given, notice that since we clearly have $\sum_{i=2}^{m}\left(\left\lfloor i \frac{n}{m}\right\rfloor-\left\lfloor(i-1) \frac{n}{m}\right\rfloor\right)=n$, the degree of the resulting polynomial must be

$$
m-1-\sum_{i=2}^{m}\left(\left\lfloor i \frac{n}{m}\right\rfloor-\left\lfloor(i-1) \frac{n}{m}\right\rfloor\right)+\left(\begin{array}{c}
m-1 \\
2
\end{array}\right)=m-1-n+\left(\begin{array}{c}
m-1 \\
2
\end{array}\right)<\left(\begin{array}{c}
m \\
2
\end{array}\right)
$$

as desired to show this polynomial to anti-symmetrize to zero.

To prove 94 we note that the equality in 24 (3), namely

$$
n a=b m+1,
$$

together with $(m, n)=(a, b)+(c, d)$, gives that

$$
n c=m d-1 .
$$

Thus since $\frac{n+1}{m}<1$

$$
\left\lfloor c \frac{n}{m}\right\rfloor-\left\lfloor(c-1) \frac{n}{m}\right\rfloor=\left\lfloor d-\frac{1}{m}\right\rfloor-\left\lfloor d-\frac{n+1}{m}\right\rfloor=(d-1)-(d-1)=0 .
$$

Likewise 95 gives 


$$
\left\lfloor(a+1) \frac{n}{m}\right\rfloor-\left\lfloor a \frac{n}{m}\right\rfloor=\left\lfloor b+\frac{n+1}{m}\right\rfloor-\left\lfloor b+\frac{1}{m}\right\rfloor=0
$$

again since $\frac{n+1}{m}<1$, completing our proof of 94 .

Finally suppose that $n=m-1$. Since in this case $\operatorname{Split}(m, m-1)=$ $(m-1, m-2)+(1,1)$ and

$$
\prod_{i=2}^{m} \frac{z_{i}}{z_{i}^{\left\lfloor i \frac{n}{m}\right\rfloor-\left\lfloor(i-1) \frac{n}{m}\right\rfloor}}=1
$$

then 92 reduces to

$$
\operatorname{Asym}_{m}\left(\left(\frac{z_{2}}{z_{1}}-(q+1 / q)+\frac{z_{m-1}}{z_{m}}\right) \prod_{i=1}^{m-2} \prod_{j=i+2}^{m}\left(z_{j}-z_{i}\right)\right)=0 .
$$

We claim that in this case we separately have

$$
\begin{gathered}
\operatorname{Asym}_{m}\left(\prod_{i=1}^{m-2} \prod_{j=i+2}^{m}\left(z_{j}-z_{i}\right)\right)=0 \\
\text { a) } \operatorname{Asym}_{m}\left(\frac{z_{2}}{z_{1}} \prod_{i=1}^{m-2} \prod_{j=i+2}^{m}\left(z_{j}-z_{i}\right)\right)=0 \\
\text { b) } \operatorname{Asym}_{m}\left(\frac{z_{m}-1}{z_{m}} \prod_{i=1}^{m-2} \prod_{j=i+2}^{m}\left(z_{j}-z_{i}\right)\right)=0 .
\end{gathered}
$$

Now 97 is immediate since the polynomial that is anti-symmetrized is of degree $\left(\begin{array}{c}m-1 \\ 2\end{array}\right)$. We will complete our proof of 91 by showing 98 a). The identity in 98 b) can be dealt with in an entirely analogous manner.

By collecting terms with respect to $z_{1}$, we have

$$
\prod_{i=1}^{m-2} \prod_{j=i+2}^{m}\left(z_{j}-z_{i}\right)=z_{3} \cdots z_{m} \prod_{i=2}^{m-2} \prod_{j=i+2}^{m}\left(z_{j}-z_{i}\right)+\sum_{k=1}^{m-2} z_{1}^{k} P_{k}\left(z_{2}, \ldots, z_{m}\right)
$$

where $P_{k}$ is a polynomial for each $k \geq 1$. It follows that for all $k \geq 1, z_{2} / z_{1}$. $z_{1}^{k} P_{k}$ is a polynomial of degree $\left(\begin{array}{c}m-1 \\ 2\end{array}\right)$ and hence they $S_{m}$-antisymmetrizes to 0 . For the only remaining term, we observe that

$$
\operatorname{Asym}_{m}\left(z_{2} / z_{1} \cdot z_{3} \cdots z_{m} \prod_{i=2}^{m-2} \prod_{j=i+2}^{m}\left(z_{j}-z_{i}\right)\right)
$$




$$
=\operatorname{Asym}_{m}\left(\frac{1}{z_{1}} z_{2} z_{3} \cdots z_{m} \operatorname{Asym}_{2, m}\left(\prod_{i=2}^{m-2} \prod_{j=i+2}^{m}\left(z_{j}-z_{i}\right)\right)\right)=0 .
$$

Since

$$
\operatorname{Asym}_{2, m}\left(\prod_{i=2}^{m-2} \prod_{j=i+2}^{m}\left(z_{j}-z_{i}\right)\right)=0
$$

holds true for the same reason we have 97 . This completes our proof.

\section{Remark 3.1}

Convincing MAPLE to deliver zero after symmetrization in 89 is not trivial even when $m$ is as small as 5 . We actually succeeded in pushing the verification of 89 for all co-prime pairs $(m, n)$ with $1 \leq n \leq m-1$ and $m \leq 7$. This given, it is worth while sketching at least what we did for $m=5$. The cases $m=6,7$ use only more elaborate versions of the same ideas.

The first step is to notice we may write

$$
\begin{aligned}
\prod_{1 \leq i<j \leq m} \Omega\left[-\frac{z_{i}}{z_{j}} M\right] & =\prod_{1 \leq i<j \leq m} \frac{\left(z_{j}-z_{i}\right)\left(z_{j}-q t z_{i}\right)}{\left(z_{j}-t z_{i}\right)\left(z_{j}-q z_{i}\right)} \\
& =\prod_{1 \leq i<j \leq m} \frac{\left(z_{j}-z_{i}\right)\left(z_{j}-q t z_{i}\right)\left(z_{i}-t z_{j}\right)\left(z_{i}-q z_{j}\right)}{\left(z_{j}-t z_{i}\right)\left(z_{j}-q z_{i}\right)\left(z_{i}-t z_{j}\right)\left(z_{i}-q z_{j}\right)} .
\end{aligned}
$$

Since the expression

$$
\prod_{1 \leq i<j \leq m}\left(z_{j}-t z_{i}\right)\left(z_{j}-q z_{i}\right)\left(z_{i}-t z_{j}\right)\left(z_{i}-q z_{j}\right)
$$

is symmetric in $z_{1}, z_{2}, \ldots, z_{m}$, it may be omitted in 89 and the Negut identity may be also be established by proving that

$$
\begin{aligned}
\operatorname{Asym}_{m}\left(\Xi_{m, n}\left[Z_{m}\right]\right. & \left(\frac{z_{c+1}}{z_{c}}-(t+q)+q t \frac{z_{a}}{z_{a+1}}\right) \\
& \left.\times \prod_{1 \leq i<j \leq m}\left(z_{j}-q t z_{i}\right)\left(z_{i}-t z_{j}\right)\left(z_{i}-q z_{j}\right)\right)=0 .
\end{aligned}
$$

Now recall that we may write, for $\frac{n}{m}<1$,

$$
\Xi_{m, n}\left[Z_{m}\right]=\prod_{i=2}^{m} \frac{z_{i}}{z_{i}^{\left\lfloor i \frac{n}{m}\right\rfloor-\left\lfloor(i-1) \frac{n}{m}\right\rfloor}} \prod_{i=1}^{m-1} \frac{1}{z_{i+1}-q t z_{i}} .
$$


Observing that the expression

$$
\mathcal{E}=\prod_{2 \leq j \leq m}\left(z_{1}-t z_{j}\right)\left(z_{1}-q z_{j}\right)
$$

is symmetric in $z_{2}, z_{3}, \ldots z_{m}$, to prove 99 we may start by anti-symmetrizing, with respect the symmetric group $S_{2, m}$, the expression

$$
\begin{aligned}
\mathcal{F}=\left(\frac{z_{c+1}}{z_{c}}\right. & \left.-(t+q)+q t \frac{z_{a}}{z_{a+1}}\right) \prod_{i=2}^{m} \frac{z_{i}}{z_{i}^{\left\lfloor i \frac{n}{m}\right\rfloor-\left\lfloor(i-1) \frac{n}{m}\right\rfloor}} \\
& \times \prod_{i=1}^{m-2} \prod_{j=i+2}^{m}\left(z_{j}-q t z_{i}\right)\left(z_{i}-t z_{j}\right)\left(z_{i}-q z_{j}\right) .
\end{aligned}
$$

We now are reduced to checking that

$$
\operatorname{Asym}_{m}\left(\mathcal{E} \operatorname{Asym}_{2, m} \mathcal{F}\right)=0 .
$$

However, we can improve on memory usage when computing $A s y m_{2, m} \mathcal{F}$. In fact, noticing that $\mathcal{F}$ is a Laurent polynomial we need only rewrite it in what we shall refer to as a normal form. More precisely this amounts to replacing $A s y m_{2, m} \mathcal{F}$ by the Laurent polynomial $\mathcal{N} \mathcal{F} \mathcal{F}$ obtained by removing from $\mathcal{F}$ all the monomials with repeated exponents and then replacing each of the remaining monomials by the rearrangement that makes the exponents decrease, multiplied by the sign of the permutation that produces that rearrangement. Since the $S_{2, m}$ anti-symmetrization of such a normalized monomial produces the same polynomial yielded by the original monomial, it follows that there is no loss in replacing $A s y m_{2, m} \mathcal{F}$ by $\mathcal{N F \mathcal { F }}$ in 100.

It turns out that the reduction in size caused by the combination of these simple tricks makes MAPLE recognize that

$$
\operatorname{Asym}_{m}\left(\mathcal{E} \operatorname{Asym}_{2, m} \mathcal{N} \mathcal{F} \mathcal{F}\right)=0
$$

at least for $m=5$. For $m=6,7$ further partial anti-symmetrizations are necessary but the basic idea is to reduce the size as much as possible within successive anti-symmetrizations.

\section{Appendix. The computation of $D_{a} D_{b}^{*}-D_{b}^{*} D_{a}$}

We should mention that the identity proved here was originally obtained using the theory of constant terms developed in [19]. What we give here is a 
completely elementary proof worked out for an audience that is unfamiliar with the above mentioned theory.

We will adopt the following convention. For $E_{i}\left[t_{1}, t_{2}, \ldots\right]$ any rational functions of the variables $t_{1}, t_{2}, \ldots$ and $P$ any symmetric polynomial, we set

$$
P^{\left(r_{1}, r_{2}, \ldots, r_{k}\right)}[X]=\left.P\left[X+E_{1} u_{1}+E_{2} u_{2}+\cdots+E_{k} u_{k}\right]\right|_{u_{1}^{r_{1}} u_{2}^{r_{2} \ldots} u_{k}^{r_{k}}} .
$$

The important property is that if

$$
P^{\left(s_{1}\right)}[X]=\left.P\left[X+E_{1} u_{1}\right]\right|_{u_{1}^{s_{1}}}
$$

then

$$
\left.P^{\left(s_{1}\right)}\left[X+E_{2} u_{2}\right]\right|_{u_{2}^{s_{2}}}=\left.P\left[X+E_{1} u_{1}+E_{2} u_{2}\right]\right|_{u_{1}^{s_{1}} u_{2}^{s_{2}}} .
$$

For $P$ a homogeneous symmetric polynomial of degree $d$ we have

$$
\begin{aligned}
D_{b}^{*} P[X] & =\left.P\left[X-\frac{\widetilde{M}}{z_{2}}\right] \Omega\left[z_{2} X\right]\right|_{z_{2}^{b}} \\
& =\left.\sum_{r_{2}=0}^{d} P^{\left(r_{2}\right)}[X]\left(\frac{1}{z_{2}}\right)^{r_{2}} \sum_{u \geq 0} z_{2}^{u} h_{u}[X]\right|_{z_{2}^{b}}=\sum_{r_{2}=0}^{d} P^{\left(r_{2}\right)}[X] h_{r_{2}+b}[X] .
\end{aligned}
$$

Thus applying 101 with $E_{1}=M$ and $E_{2}=-\widetilde{M}$ gives

$$
\begin{aligned}
D_{a} D_{b}^{*} P[X] & =\left.\sum_{r_{1}, r_{2}=0}^{d} P^{\left(r_{1}, r_{2}\right)}[X]\left(\frac{1}{z_{1}}\right)^{r_{1}} h_{r_{2}+b}\left[X+\frac{M}{z_{1}}\right] \Omega\left[-z_{1} X\right]\right|_{z_{1}^{a}} \\
& =\sum_{r_{1}, r_{2}=0}^{d} P^{\left(r_{1}, r_{2}\right)}[X]\left(\frac{1}{z_{1}}\right)^{r_{1}} \sum_{s=0}^{r_{2}+b} h_{r_{2}+b-s}[X]\left(\frac{1}{z_{1}}\right)^{s} h_{s}[M] \\
& \times\left.\sum_{u \geq 0} z_{1}^{u} h_{u}[-X]\right|_{z_{1}^{a}} \\
= & \sum_{r_{1}, r_{2}=0}^{d} P^{\left(r_{1}, r_{2}\right)}[X] \sum_{s=0}^{r_{2}+b} h_{r_{2}+b-s}[X] h_{s}[M] h_{r_{1}+s+a}[-X] .
\end{aligned}
$$

Making the summation parameter change $u=r_{1}+s+a$ gives $s=$ $u-r_{1}-a$ and the range

$$
r_{1}+a \leq u \leq r_{1}+r_{2}+a+b
$$


so 102 becomes

$$
D_{a} D_{b}^{*} P[X]=\sum_{r_{1}, r_{2}=0}^{d} P^{\left(r_{1}, r_{2}\right)}[X] \sum_{u=r_{1}+a}^{r_{1}+r_{2}+a+b} h_{r_{1}+r_{2}+a+b-u}[X] h_{u}[-X] h_{u-r_{1}-a}[M] .
$$

Similarly

$$
\begin{aligned}
D_{a} P[X] & =\left.P\left[X+\frac{M}{z_{1}}\right] \Omega\left[-z_{1} X\right]\right|_{z_{1}^{a}} \\
& =\left.\sum_{r_{1}=0}^{d} P^{\left(r_{1}\right)}[X]\left(\frac{1}{z_{1}}\right)^{r_{1}} \sum_{u \geq 0} z_{1}^{u} h_{u}[-X]\right|_{z_{1}^{a}}=\sum_{r_{1}=0}^{d} P^{\left(r_{1}\right)}[X] h_{r_{1}+a}[-X] .
\end{aligned}
$$

Thus

$$
\begin{aligned}
D_{b}^{*} D_{a} P[X] & =\left.\sum_{r_{1}, r_{2}=0}^{d} P^{\left(r_{1}, r_{2}\right)}[X]\left(\frac{1}{z_{2}}\right)^{r_{2}} h_{r_{1}+a}\left[-X+\frac{\widetilde{M}}{z_{2}}\right] \Omega\left[z_{2} X\right]\right|_{z_{2}^{b}} \\
& =\sum_{r_{1}, r_{2}=0}^{d} P^{\left(r_{1}, r_{2}\right)}[X]\left(\frac{1}{z_{2}}\right)^{r_{2}} \sum_{s=0}^{r_{1}+a} h_{r_{1}+a-s}[-X]\left(\frac{1}{z_{2}}\right)^{s} h_{s}[\widetilde{M}] \\
& \times\left.\sum_{u \geq 0} z_{2}^{u} h_{u}[X]\right|_{z_{2}^{b}} \\
& =\sum_{r_{1}, r_{2}=0}^{d} P^{\left(r_{1}, r_{2}\right)}[X] \sum_{s=0}^{r_{1}+a} h_{r_{1}+a-s}[-X] h_{s}[\widetilde{M}] h_{r_{2}+s+b}[X] .
\end{aligned}
$$

Making the summation parameter change $u=r_{1}+a-s$ gives $s=$ $r_{1}+a-u$ and the range

$$
0 \leq u \leq r_{1}+a
$$

so 103 becomes

$$
D_{b}^{*} D_{a} P[X]=\sum_{r_{1}, r_{2}=0}^{d} P^{\left(r_{1}, r_{2}\right)}[X] \sum_{u=0}^{r_{1}+a} h_{u}[-X] h_{r_{1}+r_{2}+a+b-u}[X] h_{r_{1}+a-u}[\widetilde{M}] .
$$

Now recall that

$$
\begin{aligned}
& h_{m}[M]=\frac{M}{1-t q}\left(1-(t q)^{m}\right) \quad \text { and } \\
& h_{m}[\widetilde{M}]=\frac{M}{1-t q} \frac{1-(t q)^{m}}{(t q)^{m}}=-\frac{M}{1-t q}\left(1-(t q)^{-m}\right) .
\end{aligned}
$$


We thus get

$$
\begin{aligned}
D_{a} D_{b}^{*} P[X]=\frac{M}{1-t q} & \sum_{r_{1}, r_{2}=0}^{d} P^{\left(r_{1}, r_{2}\right)}[X] \\
& \times \sum_{u=r_{1}+a}^{r_{1}+r_{2}+a+b} h_{r_{1}+r_{2}+a+b-u}[X] h_{u}[-X]\left(1-(t q)^{u-r_{1}-a}\right)
\end{aligned}
$$

and

$$
\begin{aligned}
D_{b}^{*} D_{a} P[X]=-\frac{M}{1-t q} & \sum_{r_{1}, r_{2}=0}^{d} P^{\left(r_{1}, r_{2}\right)}[X] \\
& \times \sum_{u=0}^{r_{1}+a} h_{u}[-X] h_{r_{1}+r_{2}+a+b-u}[X]\left(1-(t q)^{u-r_{1}-a}\right) .
\end{aligned}
$$

Hence

$$
\begin{aligned}
& \left(D_{a} D_{b}^{*}-D_{b}^{*} D_{a}\right) P[X]= \\
& =\frac{M}{1-t q} \sum_{r_{1}, r_{2}=0}^{d} P^{\left(r_{1}, r_{2}\right)}[X] \sum_{u=0}^{r_{1}+r_{2}+a+b} h_{r_{1}+r_{2}+a+b-u}[X] h_{u}[-X]\left(1-(t q)^{u-r_{1}-a}\right) \\
& =\frac{M}{1-t q} \sum_{r_{1}, r_{2}=0}^{d} P^{\left(r_{1}, r_{2}\right)}[X] h_{r_{1}+r_{2}+a+b}[X-X] \\
& \quad-\frac{M}{1-t q} \sum_{r_{1}, r_{2}=0}^{d} P^{\left(r_{1}, r_{2}\right)}[X](t q)^{-r_{1}-a} \sum_{u=0}^{r_{1}+r_{2}+a+b} h_{r_{1}+r_{2}+a+b-u}[X] h_{u}[-t q X] \\
& =\frac{M}{1-t q} \sum_{r_{1}, r_{2}=0}^{d} P^{\left(r_{1}, r_{2}\right)}[X] h_{r_{1}+r_{2}+a+b}[X-X] \\
& \quad-\frac{M}{1-t q} \sum_{r_{1}, r_{2}=0}^{d} P^{\left(r_{1}, r_{2}\right)}[X](t q)^{-r_{1}-a} h_{r_{1}+r_{2}+a+b}[X(1-t q)] .
\end{aligned}
$$

But now note that we may write

$$
\sum_{r_{1}, r_{2}=0}^{d} P^{\left(r_{1}, r_{2}\right)}[X](t q)^{-r_{1}-a} h_{r_{1}+r_{2}+a+b}[X(1-t q)]
$$




$$
\begin{aligned}
& =\left.\left.\frac{1}{(t q)^{a}} \sum_{r_{1}, r_{2}=0}^{d} P\left[X+M u_{1}-\widetilde{M} u_{2}\right]\right|_{u_{1}^{r_{1}} u_{2}^{r_{2}}}\left(\frac{1}{t q z}\right)^{r_{1}}\left(\frac{1}{z}\right)^{r_{2}} \Omega[z X(1-t q)]\right|_{z^{a+b}} \\
& =\left.\frac{1}{(t q)^{a}} P\left[X+\frac{M}{q t z}-\frac{\widetilde{M}}{z}\right] \Omega[z X(1-t q)]\right|_{z^{a+b}} \\
& =\left.\frac{1}{(t q)^{a}} P[X] \Omega[z X(1-t q)]\right|_{z^{a+b}} .
\end{aligned}
$$

This proves

$$
\left(D_{a} D_{b}^{*}-D_{b}^{*} D_{a}\right) P[X]=\frac{M}{1-t q} \begin{cases}\frac{1}{(t q)^{a}} h_{a+b}[X(1-t q)] P[X] & \text { if } a+b>0 \\ \left(1-\frac{1}{(t q)^{a}}\right) P[X] & \text { if } a+b=0 \\ \sum_{r_{1}+r_{2}=-(a+b)} P^{\left(r_{1}, r_{2}\right)}[X] & \text { if } a+b<0 .\end{cases}
$$

\section{References}

[1] F. Bergeron and A. M. Garsia. Science Fiction and Macdonald's Polynomials. CRM Proceedings \& Lecture Notes, American Mathematical Society, 22:1-52, 1999. MR1726826

[2] F. Bergeron, A. M. Garsia, M. Haiman, and G. Tesler. Identities and positivity conjectures for some remarkable operators in the theory of symmetric functions. Methods Appl. Anal., 6:363-420, 1999. MR1803316

[3] F. Bergeron, A. M. Garsia, E. Leven, and G. Xin. A Compositional $(k m, k n)$-Shuffle Conjecture. arXiv preprint arXiv:1404.4616. To appear in Int. Math. Res. Not., 2015.

[4] I. Burban and O. Schiffmann. On the Hall algebra of an elliptic curve, I. Duke J. Math., 161(7):1171-1231, 2012. MR2922373

[5] A. Duane, A. M. Garsia, and M. Zabrocki. A new 'dinv' arising from the two part case of the Shuffle Conjecture. J. of Alg. Comb., 37(4):683-715, 2013. MR3047015

[6] A. M. Garsia, J. Haglund, and G. Xin. Constant term methods in the theory of Tesler matrices and Macdonald polynomial operators. Annals of Comb., 1-27, 2009.

[7] A. M. Garsia and M. Haiman. Some natural bigraded $S_{n}$-Modules and q,t-Kostka coefficients. Electron. J. Combin., 3(2), 1996. MR1392509

[8] A. M. Garsia, M. Haiman, and G. Tesler. Explicit plethystic formulas for Macdonald $(q, t)$-Kostka coefficients. Séminaire Lotharingien de Combinatoire [electronic only], 42:B42m, 1999. MR1701592 
[9] A. M. Garsia, G. Xin, and M. Zabrocki. Hall Littlewood operators in the Theory of parking functions and diagonal harmonics. Int. Math. Res. Not., 6:1264-1299, 2012. MR2899952

[10] I. Gessel. Multipartite P-partitions and inner products of skew Schur functions. Contemp. Math., 34:289-301, 1984. MR0777705

[11] E. Gorsky and A. Negut. Refined knot invariants and Hilbert schemes. J. de Math. Pures et Appl., 104(3):403-435, 2015. MR3383172

[12] J. Haglund, M. Haiman, N. Loehr, J. B. Remmel, and A. Ulyanov. A combinatorial formula for the character of the diagonal coinvariants. Duke J. Math., 126:195-232, 2005. MR2115257

[13] J. Haglund, J. Morse, and M. Zabrocki. A compositional refinement of the shuffle conjecture specifying touch points of the Dyck path. Canad. J. Math., 64:822-844, 2012. MR2957232

[14] T. Hikita. Affine Springer fibers of type a and combinatorics of diagonal coinvariants. Adv. in Math., 263:88-122, 2014. MR3239135

[15] Y. Kim. A Parking Function Setting for Nabla Images of Schur Functions. FPSAC Proc. AS, 1035-1046, 2013. MR3091059

[16] I. G. Macdonald. Symmetric functions and Hall polynomials. Oxford Mathematical Monographs, New York, 2 edition, 1995. MR1354144

[17] A. Negut. The shuffle algebra revisited. Int. Math. Res. Not., rnt156, 2013. MR3283004

[18] A. Negut. Moduli of flags of sheaves on $\mathbb{P}^{2}$ and their K-theory. Alg. Geo., 2(1), 2015. MR3322196

[19] O. Schiffmann. On the Hall algebra of an elliptic curve, II. Duke J. Math., 161(9):1711-1750, 2012. MR2942792

[20] O. Schiffmann and E. Vasserot. The elliptical Hall algebra, Cherednik Hecke algebras and Macdonald polynomials. Compos. Math., 147(1):188-234, 2011. MR2771130

[21] O. Schiffmann and E. Vasserot. The elliptical Hall algebra and the equivariant K-theory of the Hilbert scheme of $\mathbb{A}^{2}$. Duke J. Math., 162(2):279-366, 2013. MR3018956

[22] G. Xin. A fast algorithm for MacMahon's partition analysis. Electron. J. Combin., 11:R58, 2014. MR2097324

[23] D. Zeilberger. A unified approach to Macdonald's root system conjectures. SIAM J. Math. Anal., 19(4):987-1013, 1988. MR0946656 
[24] D. Zeilberger. Proof of the alternating sign matrix conjecture. Electron. J. Combin., 3(2):R13, 1996. MR1392498

Francois Bergeron

DÉPARTEMEnt de MathÉmatiques

LACIM, UQAM

CANADA

E-mail address: bergeron.francois@uqam.ca

Adriano Garsia

DEPARTMENT OF MATHEMATICS

UCSD

USA

E-mail address: garsia@math.ucsd.edu

Emily Sergel Leven

DEPARTMENT OF MATHEMATICS

UCSD

USA

E-mail address: esergel@ucsd.edu

Guoce Xin

SChoOl of Mathematical SCIENCE

CAPital Normal University

PR CHINA

E-mail address: guoce_xin@163.com

Received 10 September 2014 